\title{
Review
}

\section{Power Scaling of CW Crystalline OPOs and Raman Lasers}

\author{
Soumya Sarang * and Martin Richardson
}

check for

updates

Citation: Sarang, S.; Richardson, M. Power Scaling of CW Crystalline OPOs and Raman Lasers. Photonics 2021, 8, 565. https://doi.org/ $10.3390 /$ photonics 8120565

Received: 29 October 2021

Accepted: 6 December 2021

Published: 10 December 2021

Publisher's Note: MDPI stays neutral with regard to jurisdictional claims in published maps and institutional affiliations.

Copyright: (c) 2021 by the authors. Licensee MDPI, Basel, Switzerland. This article is an open access article distributed under the terms and conditions of the Creative Commons Attribution (CC BY) license (https:// creativecommons.org/licenses/by/ $4.0 /)$.
Laser Plasma Laboratory, CREOL, The College of Optics \& Photonics, University of Central Florida, Orlando, FL 32618, USA; mcr@creol.ucf.edu

* Correspondence: soumya.sarang@ucf.edu

\begin{abstract}
Optical parametric oscillators (OPOs) and Raman lasers are two nonlinear-based laser technologies that extend the spectral range of conventional inversion lasers. Power and brightness scaling of lasers are significant for many applications in industry, medicine, and defense. Considerable advances have been made to enhance the power and brightness of inversion lasers. However, research around the power scaling of nonlinear lasers is lacking. This paper reviews the development and progress of output power of continuous-wave (CW) crystalline OPOs and Raman lasers. We further evaluate the power scalability of these two laser technologies by analyzing the cavity architectures and gain materials used in these lasers. This paper also discusses why diamond Raman lasers (DRLs) show tremendous potential as a single laser source for generating exceedingly high output powers and high brightness.
\end{abstract}

Keywords: optical parametric oscillators; diamond Raman lasers; power scaling; brightness scaling; diamond

\section{Introduction}

The wavelengths generated by inversion lasers are determined by energy level transitions in the gain material. These, and the availability of suitable efficient pumping manifolds, restrict the output of these lasers to specific bands of wavelengths, leaving "gaps" in the electromagnetic spectrum where no efficient inversion lasers have yet been demonstrated. Figure 1 illustrates such gaps in the blue region (400-440 nm), yelloworange-red wavelengths $(550-650 \mathrm{~nm})$ in the visible region, and various regions of the infrared [1,2].

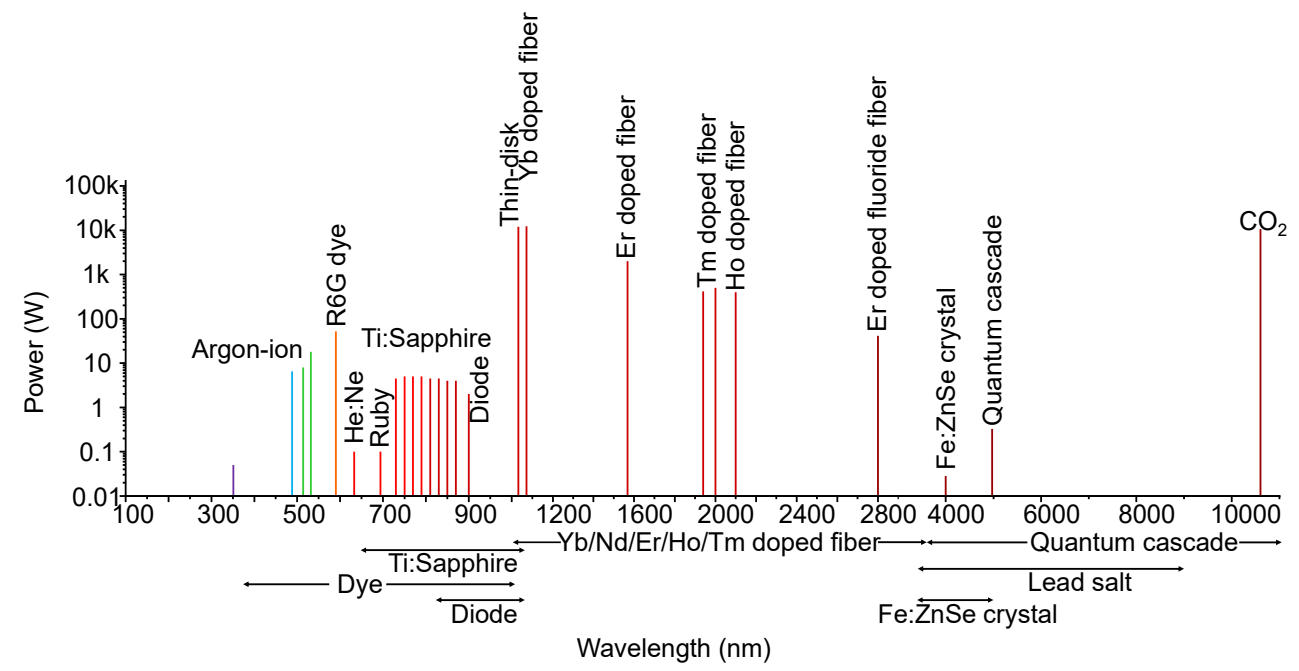

Figure 1. Wavelength and power of available inversion lasers.

Ion-lasers, Ti: Sapphire, dye, and semiconductor diode lasers are the most common commercially available continuous-wave (CW) lasers used for the visible spectral 
range [3-9]. However, the output power of single-laser sources is mostly limited to tens of watts. In near-IR spectral range from 1 to $2.5 \mu \mathrm{m}$, thin-disk lasers and rare earth dopedfiber lasers (such as ytterbium (Yb), erbium (Er), thulium (Tm), holmium (Ho)) are the prominent light sources providing extremely high powers with nearly diffraction-limited beam quality [10-17]. A Yb:YAG single-disk has delivered about $12 \mathrm{~kW}$ of CW output power at $1030 \mathrm{~nm}$ [13], whereas a single $\mathrm{Yb}$-doped fiber has achieved about $6 \mathrm{~kW}$ of output power [18]. IPG Photonics offers commercial Yb-doped single-mode fiber of about $10 \mathrm{~kW}$ at $1074 \mathrm{~nm}$ with a beam quality of $\mathrm{M}^{2}=1.05$ [19]. Despite the availability of exceedingly powerful laser sources, there are still significant voids in this spectral region, particularly the "eye-safe" wavelengths from 1.4 to $1.8 \mu \mathrm{m}$. It is also evident from the figure that there are fewer sources catering to UV and mid-IR regions. The current mid-IR light sources are quantum-cascade semiconductor lasers, lead salt lasers, lasers doped with trivalent rare-earth ions, Cr:ZnS(e) and Fe:ZnS(e) lasers [20-23]. However, these lasers are limited in their spectral tunability, output power, and low beam quality.

The lack of efficient sources with wavelengths in these regions is significant for applications in many diverse areas such as in medical laser therapy, laser guide stars, directed energy, defense, remote sensing, LIDAR, optical communications, and material processing etc., [24-28]. Many of these applications require light sources with high output power, good beam quality, and minimal divergence. For example, free-space optical-communications, LIDAR, and remote sensing applications demand broad wavelength-tunable lasers to probe a wide range of molecules with different absorption peaks. Additionally, these lasers should have high optical power, good beam quality with minimum divergence to achieve high signal to noise ratio while covering large distances. Directed energy weapons necessitate high power lasers as high as 1 to multiple- $100 \mathrm{~kW}$ depending on their operational impact [29]. Material processing applications such as cutting, welding, or drilling on metals require multi-kW output powers with moderate beam quality. On the other hand, drilling fine holes, remote welding, engraving, and micromachining require good beam quality for marking, high precision and high-value structures [30]. In many cases, these applications would benefit from sources at specific wavelengths, some of which, at present do not commercially exist. In addition to producing these inaccessible wavelengths, the sources for these applications need to be robust, stable, and amenable to power scaling.

Usually, enhancement of output power has been hindered by thermally induced effects in the gain medium such as stress, fracture, birefringence, and lensing in the laser material [31,32]. Another constraint of power scaling is preserving the brightness while elevating output power. Brightness is defined as the laser power divided by the product of beam mode area and solid angle of divergence. Higher brightness implies that the laser beam has high beam quality, minimal beam divergence, long focus field, and high power density [33]. Therefore, for applications requiring high-precision and accuracy, it is imperative that the brightness of lasers is simultaneously scaled with output power. However, in most lasers, one parameter has to be sacrificed for the improvement of the other. Thus, it is important to investigate the potential for increasing beam brightness for laser technologies.

Non-linear optics, in the form of second harmonic generation (and its derivatives of higher harmonics) [34], sum- and difference-frequency generation [35,36], parametric amplifiers [37], and stimulated Raman scattering (SRS) [38] help fill in some of the "gaps" in the spectrum, but with increased complexity and reduced efficiency [39-41]. OPOs and Raman lasers are two common sources that utilize nonlinear optical processes to access new spectral regions of interest. Sometimes, these lasers can employ other nonlinear processes such as frequency doubling and tripling, sum and difference frequency generation to access more wavelengths [42]. OPOs are attractive coherent light sources for producing narrow-linewidth, broadly tunable wavelengths from visible to the mid-IR [43-46]. Owing to thermal lensing in the gain media, these lasers were able to generate $\mathrm{CW}$ output power ranging from milliwatts to watts $[43,47]$. On the other hand, Raman lasers provide a practical alternative to OPOs as they do not require birefringent crystals or quasi-phase- 
matching conditions [48]. They are also efficient frequency converters generating high CW output powers and high brightness [49-51]. Recently, a quasi-CW DRL delivered $1.2 \mathrm{~kW}$ of output power at $1240 \mathrm{~nm}$ [52].

This review examines and compares the power scalability potential of Raman lasers with OPOs, the latter being an already mature and commercially available laser technology. We provide a short summary of the underlying gain process enabling these nonlinear frequency converters. We also analyze the cavity configurations of these lasers to evaluate the feasibility of these architectures for power scaling. The subsequent section reviews the stages of development in high-power CW OPOs and Raman lasers to identify the limiting effects in upscaling power. Furthermore, we summarize the evolution of these lasers in terms of brightness enhancement. Lastly, we will discuss why DRLs offer a strong potential to be a highly efficient and high brightness frequency converter.

\section{OPOs versus Raman Lasers}

Although OPOs and Raman lasers are driven by nonlinear optical processes, it is important to understand the basic amplification mechanism of both these lasers to have a proper comparison between them. We also discuss the merits and demerits of different types of cavity configurations in these devices. It will be clear why Raman lasers can be built with a simpler cavity design than OPOs and how certain configurations are better suited for power scaling.

\subsection{Amplification Process}

OPOs rely on parametric amplification to generate two photons-namely signal and idler, with energies lower than the pump photons incident on the nonlinear crystal placed in a resonator $[2,18,19]$. The limitations of these oscillators is the requirement of a highly intense, low-divergent, and polarized pump field $[18,20]$. Raman lasers, on the other hand, rely on stimulated scattering in which pump photons interact with optical phonons to generate Stokes photons with energy lower than the pump. The pump requirements for these lasers are not as strict as for OPOs except for the need for higher intensities. Table 1 compares the basic principles of the nonlinear gain processes of OPOs and Raman lasers.

Table 1. Comparison of OPOs and Raman lasers.

\begin{tabular}{ccc}
\hline Parameters & OPO & Raman Laser \\
\hline Amplification process & Parametric amplification & Stimulated scattering \\
Nonlinear susceptibility & $\chi^{(2)}$ & $\chi^{(3)}$ \\
Nonlinearity & Parametric & Non-parametric \\
Phase-matching & Yes & No \\
Quantum defect & No & Yes \\
\hline
\end{tabular}

Nonlinearity: Parametric oscillation is a second-order parametric nonlinear process while SRS is a third-order non-parametric nonlinear process [48]. As a consequence, conservation of energy and momentum have to be satisfied in OPOs.

Phase-matching: A drawback of OPOs is the stringent requirement of phase-matching to conserve momentum, as it is a parametric process. It is achieved either by temperature tuning or angle tuning of the nonlinear crystal, thereby increasing complexity in the laser architecture. In contrast to parametric amplification, the phase-matching is always satisfied in SRS as the medium accounts for the momentum difference of the two photons [48]. Thus, there is no necessity for angle or temperature tuning in Raman lasers, which allows for simpler cavity configurations.

Quantum-defect: In the case of OPOs, the energy of the output photons is equal to the input photon's energy, implying that no heat is deposited in the gain medium [53]. Whereas the quantum defect arises in SRS process as energy difference between the input and output fields is transferred to optical phonons resulting in heating of the crystal. 
Spatial-hole-burning free gain medium: It is evident from the amplification process of these devices that their gain dynamics is inherently different from the conventional inversion lasers. In inversion lasers, the energy is stored in the gain medium in the form of an inverted population. Whereas, the gain in these nonlinear lasers is dependent on the pump intensity and thus, no energy is stored in the gain medium [54]. A major impact of this gain mechanism is that the spatial-hole-burning phenomenon is eliminated in these devices and therefore, they can operate in single-frequency regime $[28,55,56]$.

\subsection{Cavity Configurations}

There are mainly four cavity configurations for the CW operation of OPOs as illustrated in Figure 2 [57]. The simplest configuration is a singly resonant oscillator (SRO) where the cavity is resonant for either signal or idler output wavelengths (see Figure 2a). If the cavity is resonant for both the output wavelengths, it is referred to as a doubly resonant oscillator (DRO), as shown in Figure 2b. Triply resonant oscillator (TRO) is a configuration in which all the three wavelengths-pump, signal, and idler are also resonant in the cavity (see Figure 2c). The pump for TRO is restricted to single-frequency lasers. Lastly, there is an intracavity OPO where the OPO cavity is placed inside the pump cavity (as shown in Figure $2 \mathrm{~d}$ ) to lower the lasing threshold by exploiting the high intracavity pump power.

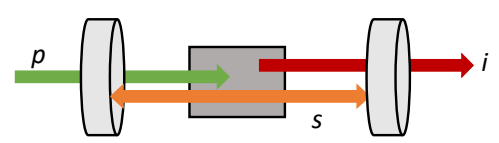

(a)

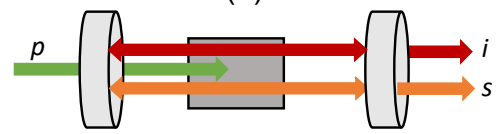

(b)

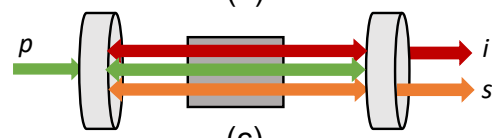

(c)

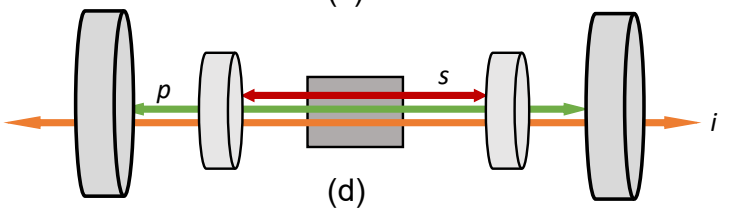

Figure 2. OPO laser (a) SRO, (b) DRO, (c) TRO, and (d) intracavity configurations; $p$, s, and i denotes pump, signal, and idler wavelengths respectively.

Although the threshold pump power required for demonstrating CW SROs is much higher than the other configurations, it provides far more practical advantages. It relaxes the stringent specifications on cavity mirror reflectivities and thus, increases the laserinduced damage threshold. Moreover, it is straightforward to satisfy the conditions of resonance and phase-matching conditions in SROs which reduces the spectral and power instabilities frequently seen in other configurations.

As shown in Figure 3, the two main Raman cavity configurations are classified as intracavity and external cavity [58]. The intracavity configuration is where the Raman gain medium is placed inside the laser cavity to resonate both the pump and Stokes wavelengths (see Figure 3a). A special case, as illustrated in Figure 3b, is self-Raman laser where the same crystal is used for laser and Raman generation. Whereas, in the external cavity configuration (see Figure 3c), the pump and Raman cavity are placed separately. Intracavity design is generally preferred over the external cavity for $\mathrm{CW}$ operation as higher intracavity pump power enables sufficient Raman gain for Stokes lasing. 


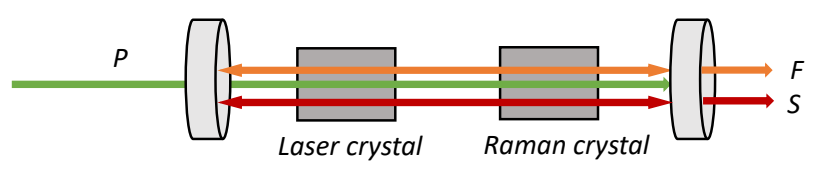

(a)
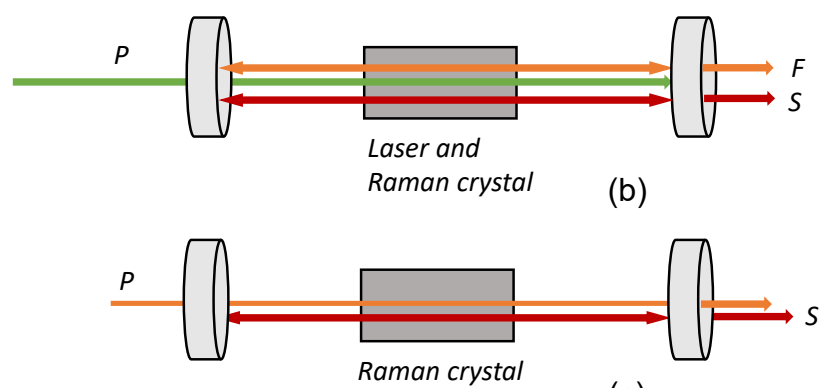

(c)

Figure 3. Raman laser (a) intracavity, (b) self-Raman, and (c) external cavity configurations; P, F, S denotes to pump, fundamental, and first Stokes respectively.

However, the design suffers from many complications. The additional coating layers on the mirrors for multiple wavelengths decreases the associated laser-induced damage threshold. Moreover, the power and frequency instabilities in the laser cavity inadvertently influence the Raman laser response. Most importantly, the thermal lensing in laser and Raman crystals drastically affect the mode stability and beam quality of Raman laser.

It is clear from the above arguments that $\mathrm{SRO}$ and the external cavity architecture are feasible for gaining output power for the OPO and Raman laser, respectively, as evident from the demonstrations detailed in Refs. [47,52,59-62].

\section{Performance of CW OPOs and Raman Lasers}

This section discusses the evolution of CW OPOs and Raman lasers and their performance characteristics such as output power, brightness, and wavelength tunability. Both these technologies have their own strong suit of parameters in which they perform better. For example, when it comes to output power and brightness, Raman lasers, especially DRLs have outperformed OPOs. On the other hand, OPOs offer commendable spectral tunability.

\subsection{Output Power of CW OPOs}

The first CW OPO demonstrated in 1968 used barium sodium niobate $\left(\mathrm{Ba}_{2} \mathrm{NaNb}_{5} \mathrm{O}_{15}\right)$ as the nonlinear gain medium pumped at $532 \mathrm{~nm}$ in a simple standing-wave cavity [63]. It achieved a conversion efficiency of $1 \%$ and the emission wavelength was tuned from $980 \mathrm{~nm}$ to $1160 \mathrm{~nm}$. Instead of the expected steady-state output, the laser emitted an output consisting of irregular spikes in the order of a few microseconds. This behavior was attributed to the fluctuations in the pump frequency and oscillator cavity length. Subsequent experiments also exhibited similar pulsed behavior except in a ring cavity configuration [64-66]. The off-angle ring cavity configuration prevented back reflections from the cavity to the pump laser, thereby enabling a steady-state output for a few milliseconds [66]. However, the improved pump and oscillator stability did not ameliorate the power characteristics. These demonstrations conveyed the need for pump sources with single-mode operation, lower noise characteristics, good beam quality, and good beam pointing stability [67]. It also demonstrated the necessity for good optical quality nonlinear materials. In short, the realization of efficient CW OPOs was hindered by the stringent specifications for pump sources and nonlinear crystals. Furthermore, the commercial availability of high-power, broad tunability, and narrow linewidth dye lasers created a major bottleneck for further development of CW OPOs, lasting for a few decades.

It was only in the mid-1980s that interest in research of CW OPOs peaked with the advent of new birefringent crystals such as $\beta-\mathrm{BaB}_{2} \mathrm{O}_{4}(\mathrm{BBO}), \mathrm{LiB}_{3} \mathrm{O}_{5}(\mathrm{LBO}), \mathrm{KTiOPO}_{4}$ 
(KTP), $\mathrm{KTiOAsO}_{4}(\mathrm{KTA})$, and $\mathrm{MgO}: \mathrm{LiNbO}_{3}$ that possess high damage threshold, favorable phase-matching, and moderate nonlinearity [57,68]. The integration of these nonlinear materials with the diode-based, solid-state crystalline, and fiber-based laser sources led to advances in the development of CW OPO technology. The initial demonstrations were realized mostly in doubly resonant/triply resonant configurations [44,69-72]. These configurations were selected owing to their capability of operating within a milliwatt pump power threshold with both signal and idler frequencies resonating in the same cavity $[44,73]$. However, the design placed strict tolerance limits on the cavity and pump frequency stabilities [74]. Since both the frequencies have to be tuned simultaneously, achieving a mode-hop-free tuning in these devices was a major challenge [75]. For the first time, SRO was demonstrated by Yang et al. in 1993 using a KTP crystal pumped by a single-frequency resonantly doubled Nd: YAG laser [76]. As expected, the threshold for the double-pass pumped laser was higher than for DROs and significant tuning was not possible. But unlike DROs, it displayed a stable CW output thereby renewing interest in SRO configuration.

By the mid-1990s, quasi-phase-matched nonlinear materials such as periodically poled lithium niobate $\left(\mathrm{LiBNO}_{3}, \mathrm{PPLN}\right)$ revived the development of SROs by bringing down the lasing threshold [77,78]. A two-mirror linear SRO using PPLN as gain material was demonstrated for the first time in 1996 [74]. It did not exhibit improvement in lasing threshold and output power. However, the key result was the ease in achieving a stable, tunable output, unlike in DROs. The same authors realized SRO using a four-mirror linear and ring configurations a few months later [79]. Similar conversion efficiencies were achieved in both the configurations, and generated output power higher than that reported previously in Ref. [74].

Table 2 lists some of the CW OPO demonstrations with configuration, total output power, signal, and idler wavelengths (emphasis given on the output power).

Table 2. Examples of CW OPOs with cavity configuration, output power, spectral ranges, and efficiency.

\begin{tabular}{|c|c|c|c|c|c|c|}
\hline $\begin{array}{c}\text { OPO } \\
\text { Based on }\end{array}$ & Configuration & $\begin{array}{c}\text { Output Power } \\
\text { (Signal + Idler) } \\
(\mathrm{W})\end{array}$ & $\begin{array}{c}\text { Signal } \\
\text { Wavelength } \\
\text { (nm) }\end{array}$ & $\begin{array}{c}\text { Idler } \\
\text { Wavelength } \\
\text { (nm) }\end{array}$ & $\begin{array}{c}\text { Efficiency }{ }^{2} \\
(\%)\end{array}$ & Ref. \\
\hline LBO & DRO & 0.103 & $494-502$ & $1320-1380$ & 9.4 & [71] \\
\hline LBO & DRO & 0.2 & 502 & 1320 & - & [70] \\
\hline PPLN & SRO & 0.9 & 953-1000 & $1160-1233$ & 64 & [45] \\
\hline PPLN & SRO & 3.55 & - & 3250 & - & [79] \\
\hline PPLN & SRO & 1 & - & $3400-3430$ & - & [80] \\
\hline MgO:sPPLT & SRO & $3.6^{1}$ & $866-978$ & 1167-1427 & 40 & [77] \\
\hline PPLN/PPKTP & DRO & $0.03-0.5$ & 656-1035 & $1096-2830$ & 69 & [43] \\
\hline MgO:PPLN & SRO & 23 & 1500 & 3600 & 77 & [61] \\
\hline MgO:PPLN & SRO & 17.5 & 1627 & 3070 & 61 & [62] \\
\hline MgO:PPLN & SRO & 30 & 1550 & 3400 & 75 & [47] \\
\hline PPLN & TRO & 0.008 & 2000-2125 & 2150-2300 & - & [46] \\
\hline PPLN & SRO & 4.4 & - & 3772 & 46.9 & [81] \\
\hline
\end{tabular}

${ }^{1}$ variable power over the spectral range; ${ }^{2}$ quantum conversion efficiency.

The advancement in singly resonant configuration led to the development of highly stable, single-frequency, high-power (up to several watts), and widely tunable OPOs [47,61,62,80,82-84]. In most of these demonstrations, thermal loading and photorefractive effect of the crystal was found to be the limiting factors for attaining higher output powers. Thermal loading was mainly attributed to the intrinsic absorption in the crystal at the pump and newly generated wavelengths. Photorefractive damage was caused by the optically induced refractive index inhomogeneities in crystals such as $\mathrm{LiNbO}_{3}, \mathrm{LiTaO}_{3}$.

In the case of SROs, achieving CW threshold necessitates extremely high intracavity power (as high as $\mathrm{kW}$ ) at signal wavelength by using high finesse cavities resulting in higher absorption and thus, heating of the nonlinear crystal [85]. The heating caused effects 
such as thermal dephasing [86-88] and thermal lensing $[85,87]$ that effectively reduced the efficiency of the laser and in some cases, damaged the nonlinear material.

Vainio et al. suggested that the effect of thermal lensing could be reduced by tightly focusing on the cavity modes [89]. Additionally, a small waist size of the beams would result in a lowering of the lasing threshold and increase pump depletion. But it could also lead to optical damage of crystals if the intracavity intensity exceeded the damage threshold. Another method used in recent demonstrations to minimize intracavity parasitic losses is by incorporating optimal output coupling leading to higher extraction efficiency and multi-watt output powers as high as $23 \mathrm{~W}[47,61,62]$. To our knowledge, the highest output power from an OPO was reported to be $30 \mathrm{~W}(19 \mathrm{~W}$ and $11 \mathrm{~W}$ of signal and idler powers at $1.55 \mu \mathrm{m}$ and $3.4 \mu \mathrm{m}$, respectively) using this method [47]. The laser operated on SLM and was stable for about $1.5 \mathrm{~h}$. However, the crystal was damaged by photorefraction when the pump power was increased further by $10 \mathrm{~W}$, thereby preventing further power scaling.

Lately, coherent beam combining (CBC) technique was proposed to enhance the output power of CW OPOs, particularly for idler wavelengths at 3-5 $\mu \mathrm{m}$. This method has been successfully implemented in high-power fiber lasers increasing their output powers to kilowatt levels [90]. The first experimental demonstration of the CBC of two difference frequency generators modules using PPLN crystals at $3.4 \mu \mathrm{m}$ was presented by Odier et al. in 2019 [91]. This demonstration was considered as the first step toward achieving the CBC of OPOs. However, the low conversion efficiency of the process limited the output power to a few milliwatts. The $\mathrm{CBC}$ of OPOs was realized experimentally for the first time in the subsequent year [92]. The results of the experiment have not been presented yet.

\subsection{Output Power of CW Raman Lasers}

$\mathrm{CW}$ operation of Raman lasers is challenging compared to the pulsed regime as high pump power (up to $100 \mathrm{~kW}$ ) is required to achieve the Raman lasing threshold. Therefore, Raman lasers were demonstrated only for specific cases such as resonant SRS [93,94], highfinesse cavities [95], and optical fibers [96-98]. Each of these cases have their respective benefits, drawbacks, and fields of applications. Gases (such as ammonia, hydrogen, methane, neon etc.,) were the preferred gain media for the initial CW Raman lasers owing to their large Raman shifts, low scattering losses, and a lower tendency for self-focusing [93-95,99]. Initial demonstrations of gaseous Raman lasers were limited in their tuning range as the pumping was restrained to atomic or molecular resonances $[93,94]$. This problem was resolved with the development of high-finesse cavities (doubly resonant at pump and Stokes wavelengths) that brought down the lasing threshold to the milliwatt level [95]. In 1998, the first non-resonant pumped Raman laser based on $\mathrm{H}_{2}$ was realized which generated $8.2 \mathrm{~mW}$ output power with $35 \%$ conversion efficiency. However, these gas media suffered from optical breakdown, have low Raman gain that required large cells leading to practical difficulties. The toxicity, high practical maintenance, and large absorption bands (in the visible and near-IR regions) prevented liquids as Raman gain media [99].

An optical fiber was used as the gain medium to achieve Raman lasing in 1976 [100]. Owing to the optical confinement provided by the small core area, optical fibers proved to be efficient frequency converters producing output powers as high as $3 \mathrm{~kW}$ and few hundreds of watts in $1 \mu \mathrm{m}$ and $1.5 \mu \mathrm{m}$ range, respectively [98,101-104]. However, the narrow optical transparency of the fiber material, small Raman shift, and the absence of resonators required for frequency doubling/tripling limited their applications [99]. These limitations led to the reawakening of interest in crystals for Raman lasers.

A major advantage of crystalline media was its high Raman gain coefficient that enabled CW Raman lasing at moderate pump powers in simple cavity configurations. Additionally, the high Raman shift, wide optical transmission, and narrow spectral broadening (higher than gases) provided a promising candidate for achieving efficient frequency conversion.

The first CW crystalline Raman laser was demonstrated in 2004. It used a barium nitrate $\left(\mathrm{Ba}\left(\mathrm{NO}_{3}\right)_{2}, \mathrm{BN}\right)$ crystal in a simple external cavity configuration pumped by a multimode laser at $532 \mathrm{~nm}$ [105]. Although the laser achieved a conversion efficiency of 5\%, 
strong thermal effects in the crystal led to roll-off in output powers at higher pump powers. Albeit the first CW Raman laser was exhibited using an external cavity architecture, the subsequent demonstrations in this temporal regime relied on intracavity architectures. This configuration was popular with a wide variety of crystals such as vanadates, tungstates (such as $\mathrm{KGd}\left(\mathrm{WO}_{4}\right)_{2}(\mathrm{KGW}), \mathrm{KY}\left(\mathrm{WO}_{4}\right)_{2}(\mathrm{KYW})$ ), molybdates etc., [106-110]. The same group demonstrated the first intracavity (self) CW Raman laser in 2005 using $\mathrm{Nd}: \mathrm{KGW}$ [106]. This laser produced about $54 \mathrm{~mW}$ of output Stokes power at $1181 \mathrm{~nm}$. In the same year, Pask et al. presented an intracavity Raman laser with Nd:YAG and KGW as laser and Raman medium, respectively [111]. It generated $800 \mathrm{~mW}$ of output power. To the best of our knowledge, among all these intracavity demonstrations, a KGW crystal recorded the highest output power of $6.1 \mathrm{~W}$ at $1176 \mathrm{~nm}$ with a conversion efficiency of $33 \%$ [108]. The output power in most of these demonstrations was limited to watt-level due to the architectural constraints on mode sizes and thermal loading management of the laser and Raman cavities and thus, prevented further power scaling [105,111].

The cavity parameters determining the efficiency of the intracavity Raman lasers were examined by Spence et al. [112]. The model elucidated the need for more complicated cavity designs (such as folded three-or four-mirrors design) to maintain cavity stability for stronger thermal lensing in the crystals. Investigations on the output spectra of intracavity lasers also revealed a broadening of the fundamental spectrum resulting in the reduction of the Raman gain coefficient [113]. This necessitated the integration of etalons in the cavity to narrow the fundamental linewidth, thereby adding new complexities to the structure. This brought the spotlight back to the external cavity architecture which was simple to design.

However, very few Raman crystals were best suited for this architecture with nonresonant pumping in the $\mathrm{CW}$ regime. Kitzler et al. reported Raman lasing in diamond using this cavity design in 2012 [114]. It exhibited an output power of $10.1 \mathrm{~W}$ with a conversion efficiency of $32 \%$ which was a six-fold improvement from $5 \%$ efficiency reported in ref. [105]. Over recent years, many experiments were conducted with diamond to achieve newer wavelengths $[25,50,115]$, single-longitudinal-mode (SLM) operation [28], and enhanced output power $[52,116]$.

Within two years after the first demonstration, researchers were able to increase the output power of a DRL by at least ten times [60]. For this demonstration, a Nd:YAG pump delivering $370 \mathrm{~W}$ at $1064 \mathrm{~nm}$ in $250 \mu$ s pulses at $40 \mathrm{~Hz}$ was used. The external-cavity quasi-CW DRL generated an output power of $108 \mathrm{~W}$ from $322 \mathrm{~W}$ of pump power. Although it was pumped in a quasi-CW temporal regime, the steady-state thermal gradients were established within $100 \mu$ s from the start of the pump pulse-width. Therefore, the DRL was considered to be operating in CW regime after the first $100 \mu \mathrm{s}$ of the pump laser. A year later, the output power was elevated to $381 \mathrm{~W}$ with a conversion efficiency of $61 \%$ [116]. The maximum output power was limited by available pump power. Although a cavity instability due to strong thermal lensing was predicted, no output power roll-off and beam quality degradation were observed indicating the development of weaker thermal lensing in the crystal than calculated.

By exploiting spatial-hole-burning-free nature of Raman gain medium, an SLM operation was achieved in a standing-wave DRL with output power of $4 \mathrm{~W}$ [28]. However, the laser displayed power and mode instabilities at higher output powers. Upon investigating the reasons for this behavior, the coupling between the output power and cavity length was found to be hindering SLM at higher powers. The heating of diamond owing to quantum defect and intrinsic absorption led to altering of the cavity length via thermal expansion and thermo-optic effect. This demonstration was the first evidence of thermal effects in a DRL. However, these thermal problems did not impede the efficiency and beam quality of the device.

By 2019, a quasi-steady-state DRL reported kilowatt level of output power [52]. This laser produced a maximum output power of $1.2 \mathrm{~kW}$ with $53 \%$ conversion efficiency. These are other noteworthy observations made in the demonstration: (a) A thermal roll-off was seen at the maximum output power of $1.23 \mathrm{~kW}$; (b) an increase in the output beam quality 
with increasing Stokes powers. For the first time, these observations indicated the presence of thermal lensing in diamond affecting output beam profile. It must be noted that the crystal was not actively cooled in the experiment. Subsequently, to estimate thermal lensing in diamond, the output beam propagation parameters were simulated as a function of Stokes output power using ABCD matrix analysis [117]. The modeled results were in agreement with the experimental observations. The improvement in the Stokes beam quality parameter $\left(\mathrm{M}^{2}\right)$ with increasing Stokes power was attributed to the development of a positive thermal lens in diamond. Furthermore, the discrepancy between the calculated and experimental thermal lens strength in previous experiments was corrected by adding a factor that included the mismatch of pump and Stokes mode sizes. By introducing this factor, the overestimation of the thermal lens and the negligible effect of lensing on the output power or spatial beam profile in ref. [116] were also explained. As the radius of the Stokes spot was 2.75 times larger than the pump spot size, the corrected thermal lens strength was 16 times smaller than the calculated one. Thus, the thermal lens in diamond was much more moderate than predicted. Table 3 summarizes some of the CW crystalline Raman lasers with cavity configurations and output characteristics.

Table 3. Examples of several CW Raman lasers with performance characteristics.

\begin{tabular}{|c|c|c|c|c|c|c|}
\hline Raman Lasers Based on & Configuration & $\begin{array}{l}\text { Output } \\
\text { Power }\end{array}$ & $\begin{array}{c}\text { Wavelength } \\
(\mathrm{nm})\end{array}$ & $\begin{array}{c}\text { Crystal Length } \\
(\mathrm{mm})\end{array}$ & $\begin{array}{l}\text { Efficiency } \\
(\%)\end{array}$ & Ref. \\
\hline $\mathrm{Nd}^{3+}: \mathrm{KGW}$ & Self-Raman & $54 \mathrm{~mW}$ & 1181 & 40 & 2.6 & [106] \\
\hline KGW & Intracavity & $800 \mathrm{~mW}$ & 1176 & 50 & 4 & [111] \\
\hline Diamond & Intracavity & $200 \mathrm{~mW}$ & 1240 & 3.3 & - & [118] \\
\hline $\mathrm{PbWO}_{4}$ & Intracavity & $178 \mathrm{~mW}$ & 1177 & 50 & 8 & [119] \\
\hline KGW & Intracavity & $1.56 \mathrm{~W}$ & 1176 & 25 & - & [120] \\
\hline Diamond & Intracavity & $4.4 \mathrm{~W}$ & 1228 & 6.5 & 14.2 & [121] \\
\hline $\mathrm{SrWO}_{4}$ & Intracavity & $2.23 \mathrm{~W}$ & 1179.5 & 46 & 10.5 & [109] \\
\hline $\mathrm{BaWO}_{4}$ & Intracavity & $3.36 \mathrm{~W}$ & 1180 & 30 & 13.2 & [122] \\
\hline $\mathrm{Nd}: \mathrm{GdVO}_{4}$ & Self-Raman & $4.1 \mathrm{~W}$ & 1173 & 20 & 11.2 & [107] \\
\hline $\mathrm{YVO}_{4} / \mathrm{Nd}: \mathrm{YVO}_{4} / \mathrm{YVO}_{4}$ & Self-Raman & $5.3 \mathrm{~W}$ & 1176 & 20 & 20 & [108] \\
\hline $\mathrm{SrMoO}_{4}$ & Intracavity & $2.18 \mathrm{~W}$ & 1173.5 & 20.5 & 8.7 & [110] \\
\hline KGW & Intracavity & $6.1 \mathrm{~W}$ & $1140-1155$ & 30 & 33 & [123] \\
\hline Diamond & Intracavity & $5.1 \mathrm{~W}$ & 1217 & 6.5 & 28 & [123] \\
\hline Yb:KGW & Self-Raman & $1.7 \mathrm{~W}$ & 1099.6 & 6 & 21.8 & [124] \\
\hline $\mathrm{Nd}: \mathrm{YVO}_{4}$ & Self-Raman & $1.8 \mathrm{~W}$ & 1176 & 22 & - & [125] \\
\hline $\mathrm{Yb}: \mathrm{KGW}$ & Intracavity & $4.5 \mathrm{~W}$ & 1096 & 4 & 33 & [126] \\
\hline $\mathrm{Ba}\left(\mathrm{NO}_{3}\right)_{2}$ & External cavity & $164 \mathrm{~mW}$ & 543 & 68 & 5 & [105] \\
\hline Diamond & External cavity & $10.1 \mathrm{~W}$ & 1240 & 9.5 & 32 & [114] \\
\hline KYW & External cavity & $2.7 \mathrm{~W}$ & 1158 & 50 & 2 & [127] \\
\hline KGW & External cavity & $6.1 \mathrm{~W}$ & 1320 & 30 & 33 & [51] \\
\hline Diamond & External cavity & $14 \mathrm{~W}$ & 1240 & 8 & 38 & {$[28]$} \\
\hline Diamond & External cavity & $7 \mathrm{~W}$ & $1485^{1}$ & 8 & 21 & [115] \\
\hline Diamond & External cavity & $108 \mathrm{~W}$ & 1240 & 8 & 34 & [60] \\
\hline Diamond & External cavity & $114 \mathrm{~W}$ & $1485^{1}$ & 8 & 44 & [128] \\
\hline Diamond & External cavity & $154 \mathrm{~W}$ & 1240 & 8 & 50.5 & [116] \\
\hline Diamond & External cavity & $389 \mathrm{~W}$ & 1240 & 8 & 52.5 & [129] \\
\hline Diamond & External cavity & $1.2 \mathrm{~kW}$ & 1240 & 8.6 & 53 & [52] \\
\hline
\end{tabular}

${ }^{1}$ Second Stokes generation. 


\subsection{Brightness Beam Enhancement of OPOs and Raman Lasers}

As mentioned earlier, brightness is an important parameter to be considered along with power scaling. The output beam brightness is evaluated according to the relation [130],

$$
\mathrm{B}=\frac{\mathrm{P}}{\lambda^{2} \mathrm{M}_{\mathrm{x}}^{2} \mathrm{M}_{\mathrm{y}}^{2}}
$$

where $\mathrm{P}, \lambda$, and $\mathrm{M}_{\mathrm{x}}^{2}, \mathrm{M}_{\mathrm{y}}^{2}$ denote the output power, wavelength, parameters of beam profile, respectively.

The efficiency of OPOs depends on the pump beam characteristics. Most of the gain crystals (such as LBO, BBO) have narrow acceptance angles, which make beam divergence a critical issue for the successful operation of OPOs [131]. Mode-matching between the pump and cavity, and transverse mode competition also affects the conversion process. Moreover, pump beam requirements for specific laser configurations are more stringent than in others. For example, DROs are more sensitive to pump laser fluctuations and mechanical instabilities. Due to these complications, most of the CW OPOs generating high output powers were pumped by the near-diffraction-limited beam $[47,62,132]$. As a result, there are not many OPO demonstrations for beam brightness enhancement except one in which a pump beam of $\mathrm{M}^{2}=5.5$ produced $1.1 \mathrm{~W}$ of idler output with a beam quality $\mathrm{M}^{2}=3.8$ [133]. A nanosecond pulsed OPO exhibited an output beam quality of $\mathrm{M}^{2}=1.7$ from a pump laser with a beam quality of $\mathrm{M}^{2}=29$ using a high magnification unstable confocal resonator [134].

On the other hand, Raman lasers are known as converters of highly aberrated pump beams into spatially clean output beams via the "Raman beam cleanup (RBC)" mechanism of the SRS process. [135]. This mechanism was seen initially in Raman gas amplifiers that produced distortion-free amplification of the highly-divergent rare-gas excimer lasers [135-137]. With the availability of high-quality crystals, solid-state Raman lasers became efficient brightness converters. As expected, most of the early CW demonstrations were conducted in intracavity and self-Raman architectures [106,122,138-142]. A self-Raman Nd:KGW laser reported in 2005 showed an output beam quality of $\mathrm{M}^{2}<1.2$ when pumped by a diode laser having a beam quality of $\mathrm{M}^{2}=39$ [106]. Another selfRaman Nd: $\mathrm{YVO}_{4}$ laser converted the pump beam quality of $\mathrm{M}^{2}=42$ to a Stokes output of $\mathrm{M}^{2}<1.4$ [141]. The significance of this mechanism can be understood by the beam quality measurements reported in a self-Raman laser in Ref. [138]. The measurements showed an increase in the $\mathrm{M}^{2}$ value of the fundamental beam, which deteriorated after the onset of Raman amplification from 8.3 to 14 (at maximum pump power). The reason for this exacerbation of the beam quality was the additional heating of the crystal owing to the SRS process. Nevertheless, the output Stokes $\mathrm{M}^{2}$ value remained at 3 thereby proving the cleanup effect of the Raman process. Similar results were observed in intracavity configurations using KGW and diamond crystals, respectively, pumped by semiconductor disk lasers (SDLs) $[139,140]$. The SDL beam quality worsened in both cases after attaining the Raman lasing threshold. Concurrently, the RBC ameliorated the beam quality of the Stokes output. A comparative study on the performance of diamond and KGW crystals in an intracavity Raman configuration was carried out by Savitski et al. [123]. Although KGW produced a slightly higher output power than diamond, it generated significantly poor beam quality than diamond. The DRL produced an output beam quality of $\mathrm{M}^{2}=1.1 \times 1.2$, whereas KGW-based Raman laser produced output $\mathrm{M}^{2}=5 \times 6$, when pumped by an $\mathrm{Nd}$ :YLF laser with $\mathrm{M}^{2}=14 \times 19$. The stronger thermal aberrations in the KGW crystal were predicted to be the cause for this observation.

Consequently, recent studies on brightness enhancement have employed the external cavity architecture to determine its viability for generating high power brightness beams. Zhenxu et al. measured the beam enhancement factor (BEF) for a quasi-CW DRL pumped at $1064 \mathrm{~nm}$ by the low-quality beam of varying $\mathrm{M}^{2}$ values for both first and second Stokes 
wavelengths $[59,143]$. BEF is defined as the ratio of the output brightness to the pump brightness. It is calculated according to the relation [143],

$$
\mathrm{BEF}=\eta_{\mathrm{e}}\left[\frac{\lambda_{\mathrm{p}}}{\lambda_{\mathrm{s}}}\right]^{2}\left[\frac{\mathrm{M}_{\mathrm{p}}^{2}}{\mathrm{M}_{\mathrm{s}}^{2}}\right]^{2},
$$

where, $\eta_{e}, \lambda_{p}$, and $\lambda_{s}, M_{p}^{2}$ and $M_{s}^{2}$ are the power conversion efficiency, pump and Stokes wavelengths, and beam quality parameter for pump and Stokes beams, respectively. The laser converted $453 \mathrm{~W}$ pump power at $\mathrm{M}^{2}=7.3$ to $180 \mathrm{~W}$ first Stokes output power having $\mathrm{M}^{2}=1.1$ [59]. A BEF of 12.7 was calculated. In the case of second-order Stokes, $302 \mathrm{~W}$ output power with an $\mathrm{M}^{2}=1.1$ was obtained from $823 \mathrm{~W}$ pump power with $\mathrm{M}^{2}=6.4$ providing a BEF of 6 [143]. The recent $1.2 \mathrm{~kW}$ quasi-CW DRL demonstration in [52] measured a $\mathrm{BEF}$ of 56 that is the largest reported for any crystalline Raman laser. The laser converted the pump beam quality of $\mathrm{M}^{2}=15$ to output beam quality of $\mathrm{M}^{2}=2.95$. Consequently, the output beam quality at the maximum pump power improved to $\mathrm{M}^{2}=1.25$ owing to thermal lensing in the gain crystal.

\subsection{Wavelength Tuning of OPOs and Raman Lasers}

One of the main attractions of OPOs is their exceptionally broad, continuous wavelength tunability (from visible to near-IR to mid-IR, to THz) [43-46]. They are widely popular as coherent sources for mid-IR wavelengths. An example is the demonstration of a doubly resonant $\mathrm{OPO}$ with signal and idler outputs covering wavelengths from 0.66 to $1.035 \mu \mathrm{m}$ and 1.096 to $2.830 \mu \mathrm{m}$, respectively [43]. The tunability is achieved by either varying the pump wavelength or the phase-matching conditions of the nonlinear medium. The latter is realized through changing the temperature, orientation, or poling periods of the crystal [144].

However, the wide tunability of OPOs comes at the cost of reduced spectral brightness. Spectral brightness is defined as the beam brightness within a spectral width. It is a significant laser parameter to be considered for applications requiring narrow-linewidth sources such as spectroscopy, remote-sensing, etc.

On the other hand, Raman lasers have successfully achieved a wide interval of emission wavelengths from ultraviolet to the near-IR range [145-147]. Recently, $22 \mathrm{~W}$ at $589 \mathrm{~nm}$ and $38 \mathrm{~W}$ at $620 \mathrm{~nm}$ of output powers were generated in two separate cases of intracavity frequency doubling in quasi-CW external cavity DRLs [25,50]. But sources catering to the mid-IR region are still sparse. Apart from varying the pump wavelength and optical frequency mixing, these devices also achieve wavelength diversification through the "Raman cascading" process [127,128]. Raman cascading is referred to as the generation of successive higher order Stokes wavelengths when the intensity of the preceding Stokes order becomes sufficiently high [99].

\section{Material Properties of OPO and Raman Crystals}

In order to understand why specific crystalline nonlinear lasers worked much better than others, we need to investigate further the material properties of the gain media used.

The performance of OPOs depends on nonlinear optical (NLO) coefficients, transparency range and optical damage thresholds. In order to bring the $\mathrm{CW}$ lasing threshold to acceptable powers, a crystal with a large gain coefficient is required that can induce substantial nonlinearity at relatively low pump powers. It is also necessary that the crystal is highly transparent at the pump and output wavelengths. Moreover, a high optical damage threshold is essential for the crystal when scaling at higher powers.

The most popular crystal of choice for $\mathrm{CW}$ operation of OPOs is lithium niobate $\left(\mathrm{LiNbO}_{3}, \mathrm{LN}\right)$ owing to its large nonlinearity of $17.6 \mathrm{pm} / \mathrm{V}$ and wide acceptance angle in quasi-phase matched condition. However, its use in OPOs was reduced, as its low optical damage threshold and higher sensitivity to photorefractive damage adversely affected the performance of the lasers [148]. A solution to increase photorefractive damage was to 
heat the crystal but it led to a change in phase-matching, thereby complicating the cavity setup. Another method to eliminate the damage was by doping the crystal with $\mathrm{MgO}$ [148]. However, doping affected the crystal quality.

The development of periodically poled lithium niobate (PPLN) provided advantages over LN for OPOs. It enabled high gain, large effective nonlinear coefficient, and no spatial walk-off. It also made the crystal less susceptible to photorefractive damage. However, its coercive field is $21 \mathrm{kV} / \mathrm{mm}$, which limited the crystal's thickness to approximately $0.5 \mathrm{~mm}$ [149].

Borate-based crystals such as $\beta$-barium borate $\left(\mathrm{BaB}_{2} \mathrm{O}_{4}, \mathrm{BBO}\right)$ and lithium triborate $\left(\mathrm{LiB}_{3} \mathrm{O}_{5}, \mathrm{LBO}\right)$ were most commonly used for OPOs because of their moderate nonlinear coefficient, mechanical hardness, chemical stability, and high optical damage threshold $[150,151]$. They transmitted far into the ultraviolet spectrum than any other crystals. Furthermore, these crystals do not suffer from photorefractive damage. LBO provided an additional benefit over $\mathrm{BBO}$ as it enabled temperature tuning for non-critical phase matching resulting in a simple cavity alignment. Moreover, it has a smaller spatial walk-off angle. However, due to their lower nonlinear coefficients compared to other crystals, they were preferred for pulsed operation.

Potassium titanyl phosphate $\left(\mathrm{KTiOPO}_{4}, \mathrm{KTP}\right)$ is another commonly used gain crystal. It has a high nonlinear coefficient of $16.9 \mathrm{pm} / \mathrm{V}$, a high optical damage threshold, and lower sensitivity to photorefractive effect $[152,153]$. It has a wide transparency range from ultraviolet to the mid-IR range with strong absorption at 2.8 and $3.4 \mu \mathrm{m}$. Moreover, another benefit of using the crystal is the easy attainment of the phase-matching through temperature tuning. Moreover, its low coercive field of $2 \mathrm{kV} / \mathrm{mm}$ allowed poling of thicker crystals [153]. Thus, KTP crystals of several millimeters (as large as $3 \mathrm{~mm}$ ) are suitable for poling and consequently higher power generation [154]. Table 4 lists some important properties of popular nonlinear materials used for OPOs.

Table 4. Comparison of material properties of OPO crystals.

\begin{tabular}{|c|c|c|c|c|}
\hline Properties at Room Temperature & LBO [151] & LN [155] & KTP $[152,153]$ & BBO [151] \\
\hline \multirow{2}{*}{$\begin{array}{c}\text { Crystal structure } \\
\text { Optical transparency } \\
(\mu \mathrm{m})\end{array}$} & Orthorhombic & Trigonal & Orthorhombic & Trigonal \\
\hline & $0.16-2.6$ & $0.33-5.5$ & $0.35-4.5$ & $0.19-3.5$ \\
\hline NLO coefficients ${ }^{1}(\mathrm{pm} / \mathrm{V})$ & $\begin{array}{l}1.02 \\
0.057 \\
-0.95\end{array}$ & $\begin{array}{l}5.7 \\
5.3 \\
17.6\end{array}$ & $\begin{array}{c}1.9 \\
3.6 \\
2.5 \\
4.4 \\
16.9\end{array}$ & $\begin{array}{c}2.3 \\
-0.16\end{array}$ \\
\hline Thermal conductivity $(\mathrm{W} / \mathrm{mK})$ & 3.5 & 5 & $\begin{array}{c}2, \\
3, \\
3.3^{2}\end{array}$ & 1.2 \\
\hline $\begin{array}{l}\text { Thermal expansion coefficient }{ }^{2} \\
\qquad\left(10^{-5} \mathrm{~K}^{-1}\right)\end{array}$ & $\begin{array}{c}10.8 \\
-8.8 \\
3.4\end{array}$ & $\begin{array}{l}0.2 \\
0.22\end{array}$ & $\begin{array}{l}1.1 \\
0.9 \\
0.06\end{array}$ & $\begin{array}{l}0.4 \\
3.6\end{array}$ \\
\hline $\begin{array}{l}\text { Thermo-optic coefficient } \\
\qquad\left(10^{-6} \mathrm{~K}^{-1}\right)\end{array}$ & $\begin{array}{l}-9.3 \\
-13.6\end{array}$ & $\begin{array}{c}-0.87 \\
39.1\end{array}$ & $\begin{array}{l}11 \\
13 \\
16\end{array}$ & $\begin{array}{l}-9.3 \\
-16.6\end{array}$ \\
\hline
\end{tabular}

\footnotetext{
${ }^{1}$ values for different susceptibility tensor elements $\mathrm{d}_{\mathrm{il}} ;{ }^{2}$ values for principal axes.
}

In addition to the desirable properties discussed in OPO gain crystals, a Raman gain crystal should also possess good thermal properties. This is due to the fact that SRS is an inelastic nonlinear process and hence, there is heat deposition in the medium.

The initial popular choices for Raman crystals were barium nitrate, double tungstate crystals (such as KGW, KYW). Barium nitrate was selected as the gain medium for the firstever CW external cavity Raman laser owing to its very high Raman gain coefficient [105]. 
But it has many disadvantages. It is hygroscopic and has a moderate optical damage threshold [156]. Furthermore, its low thermal conductivity limited the conversion efficiency of Raman lasers.

On the other hand, the thermal conductivity and transparency range of double tungstate crystals is better than barium nitrate. Additionally, its mechanical hardness and high optical damage threshold promote its use in Raman lasers. But it has a low Raman gain coefficient which constricts its implementation to mostly CW intracavity configuration. Moreover, the presence of several Raman modes also adversely affected the CW laser efficiency [127].

Another crystal that has a Raman gain coefficient comparable with barium nitrate is diamond. It possesses intrinsically extraordinarily high thermal conductivity, low thermal expansion, and high levels of hardness leading to high optical damage thresholds [54]. The combination of high gain coefficient and high damage threshold made it an outstanding candidate for realizing external cavity configurations.

With high optical transmissivity in the UV, visible, and mid-IR, its pure crystalline nature leads to low densities of microscopic scattering centers within the material resulting in low optical absorption losses, low birefringence and, unlike most doped oxide laser materials, high degrees of optical uniformity $[49,118]$. High quality diamond surfaces have been used for a number of industrial processes with the new availability of bulk single-crystalline diamond [157]. This form of diamond, fabricated by chemical vapor deposition (CVD) is the bedrock for the development of DRLs [158].

The primary challenge for using this crystal was its high birefringence and optical absorption [118]. Despite diamond being an optically isotropic crystal, it is susceptible to birefringence owing to the strain from grown-in defects during the CVD process [158,159]. Nitrogen impurities are responsible for optical absorption. The new advances in CVD technology led to the synthesis of high-optical-quality single-crystal diamonds with ultralow birefringence and absorption coefficient. The Raman-grade diamond crystals manufactured by Element Six Ltd. have nitrogen impurities and absorption coefficients of approximately $20-40 \mathrm{ppb}$ and $0.001-0.004 \mathrm{~cm}^{-1}$ (at $1064 \mathrm{~nm}$ ), respectively [158]. It has a birefringence of typically $<10^{-5}$ (in the visible and near-infrared range) perpendicular to the growth direction.

Table 5 summarizes some important properties of popular Raman crystals.

Table 5. Comparison of material properties of Raman crystals [54].

\begin{tabular}{|c|c|c|c|c|}
\hline $\begin{array}{c}\text { Properties at Room } \\
\text { Temperature }\end{array}$ & Diamond & $\mathbf{B N}$ & KGW & KYW [160,161] \\
\hline \multirow{2}{*}{$\begin{array}{l}\text { Crystal structure } \\
\text { Optical transparency } \\
(\mu \mathrm{m})\end{array}$} & Cubic & Cubic & Monoclinic & Monoclinic \\
\hline & $0.23-100$ & $0.3-1.8$ & $0.3-5$ & $0.34-5.5$ \\
\hline Raman shift $\left(\mathrm{cm}^{-1}\right)$ & 1332.3 & 1047.3 & $\begin{array}{r}901 \\
768^{1}\end{array}$ & $\begin{array}{r}905 \\
765^{1}\end{array}$ \\
\hline $\begin{array}{l}\text { Raman gain coefficient } \\
(\mathrm{cm} / \mathrm{GW}, @ 1064 \mathrm{~nm})\end{array}$ & 10 [162] & 11 & $\begin{array}{r}3.3, \\
4.4^{1}\end{array}$ & $\begin{array}{l}3.6, \\
3.6^{1}\end{array}$ \\
\hline $\begin{array}{l}\text { Raman linewidth } \\
\text { FWHM }\left(\mathrm{cm}^{-1}\right)\end{array}$ & 1.5 & 0.4 & $\begin{array}{l}7.8^{\prime} \\
5.9^{1}\end{array}$ & $6-8$ \\
\hline $\begin{array}{l}\text { Thermal conductivity } \\
\qquad(\mathrm{W} / \mathrm{mK})\end{array}$ & 2000 & 1.2 & $\begin{array}{l}2.6 \\
3.8 \\
3.4^{2}\end{array}$ & $3.3^{3}$ \\
\hline $\begin{array}{l}\text { Thermal expansion } \\
\text { coefficient }\left(10^{-6} \mathrm{~K}^{-1}\right)\end{array}$ & 1.1 & 13 [163] & $1.6-8.5[164]$ & $2-8.5[164]$ \\
\hline $\begin{array}{l}\text { Thermo-optic } \\
\text { coefficient }\left(10^{-6} \mathrm{~K}^{-1}\right)\end{array}$ & 15 & 20 & 0.4 & 0.4 \\
\hline
\end{tabular}

${ }^{1}$ values for two main Raman phonon modes; ${ }^{2}$ values for three principal axes. ${ }^{3}$ averaged over three principal axes. 


\section{Discussion: Power and Brightness Scalability of DRLs}

It is apparent from this review that thermal lensing in the crystal is a limiting factor for power and brightness scaling of both nonlinear laser technologies even though the causes of heating are distinct.

In case of OPOs, the thermal effects originate from the absorption of high intracavity signal power or absorption at the idler wavelengths [85]. In addition, for CW operation in OPOs, the thermal load is intense as the circulating power in the cavity generally exceeds the kilowatt level, even when generating low output powers. These effects lead to temperature gradients across the crystal resulting in other detrimental effects such as dephasing, focusing, aberrations, and in extreme cases material fracture [165]. On the other hand, quantum defect is the major contributor, other than parasitic crystal absorption, to thermal lensing in Raman lasers. Consequently, the thermal properties of the gain material are a decisive parameter for pursuing power gains.

Figure 4 compares the values of thermal conductivity of commonly used OPO and Raman gain crystals.

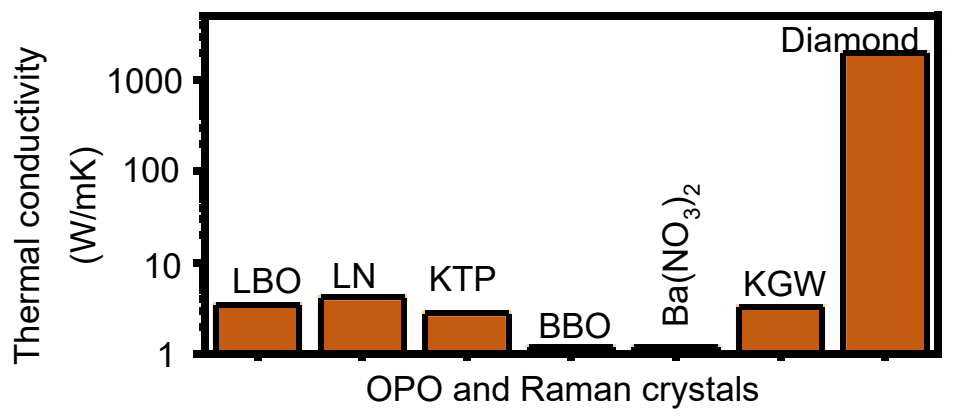

Figure 4. Thermal conductivity of common OPO and Raman gain crystals.

The thermal conductivity of diamond is $2000 \mathrm{~W} / \mathrm{mK}$, which is about three orders of magnitude higher than other gain materials as is evident from the figure. The effective focal length of thermal lens for a nonlinear crystal is expressed as,

$$
\mathrm{f}=\frac{\pi K \omega^{2}}{\alpha \mathrm{P}\left(\frac{\mathrm{dn}}{\mathrm{dT}}\right) \mathrm{L}_{\mathrm{c}}},
$$

where $K, \omega, \alpha, P, d n / d T$, and $L_{c}$ are the thermal conductivity, signal spot size, absorption coefficient, intracavity signal power, thermo-optic coefficient, and crystal length, respectively. In the case of Raman lasers, quantum defect should also be included. Therefore, $\alpha \mathrm{PL}_{\mathrm{C}}$ in Equation (2) is replaced by $\mathrm{P}_{\mathrm{dep}}$ which is defined as,

$$
\mathrm{P}_{\mathrm{dep}}=\left(\frac{\lambda_{\mathrm{s}}}{\lambda_{\mathrm{p}}}-1\right) \mathrm{P}_{\mathrm{gen}}^{\mathrm{s}}+\mathrm{P}_{\mathrm{int}}^{\mathrm{s}} \alpha \mathrm{l}_{\mathrm{R}},
$$

where $\mathrm{P}_{\text {dep }}, \lambda_{\mathrm{s}}, \lambda_{\mathrm{p}}, \mathrm{P}_{\text {gen }}^{\mathrm{s}}, \mathrm{P}^{\mathrm{s}}$ int , and $\mathrm{l}_{\mathrm{R}}$ represent deposited power, Stokes and pump wavelengths, generated Stokes power, intracavity Stokes power, and length of Raman crystal, respectively. The first and second term in Equation (3) corresponds to quantum defect and impurity absorption.

For constant power deposited in the crystal, crystal length, and spot size, the thermal focal length is given by the expression,

$$
f \propto \frac{K}{\left(\frac{d n}{d T}\right)} .
$$

So, if we compare thermal conductivity and thermo-optic coefficients of LN and diamond - two crystals that have demonstrated the highest power in OPOs and Raman 
lasers respectively, the focal length of the thermal lens for diamond is calculated to be about thousand times larger than for LN. In other words, it is highly likely that the thermal effects will exacerbate the performance of LN-based nonlinear lasers much earlier than for the diamond-based ones.

Let us now look into the progress in output power of nonlinear laser technologies (compiled in Tables 2 and 3) over the past three decades, illustrated in Figure 5. Even though the development of solid-state (except diamond) Raman lasers started more than a decade later compared to the OPOs, these lasers generated output powers similar to the OPOs. Interestingly DRLs, which were first demonstrated almost two decades later, have shown remarkable growth compared to others, stretching the powers to kilowatt level within a decade. Meanwhile, the output powers of OPOs and non-diamond Raman lasers are yet to approach even hundreds of watts; restricted by thermal lensing in the gain materials. The only other technology competitive enough to pursue such power gains is the fiber-based Raman lasers [98,101-104,166-173], as evident from the figure.

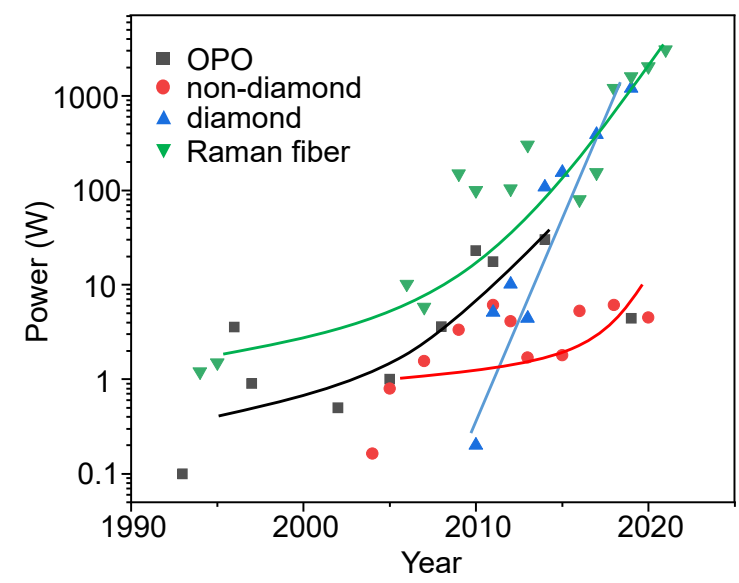

Figure 5. Evolution of the output power of CW nonlinear lasers over the past 30 years. Solid line denotes the power trend over the years.

As already discussed, power enhancement also brings in an additional requirement of good beam quality. The brightness of the nonlinear lasers over the past decade is calculated according to Equation (1), and is illustrated in Figure 6.

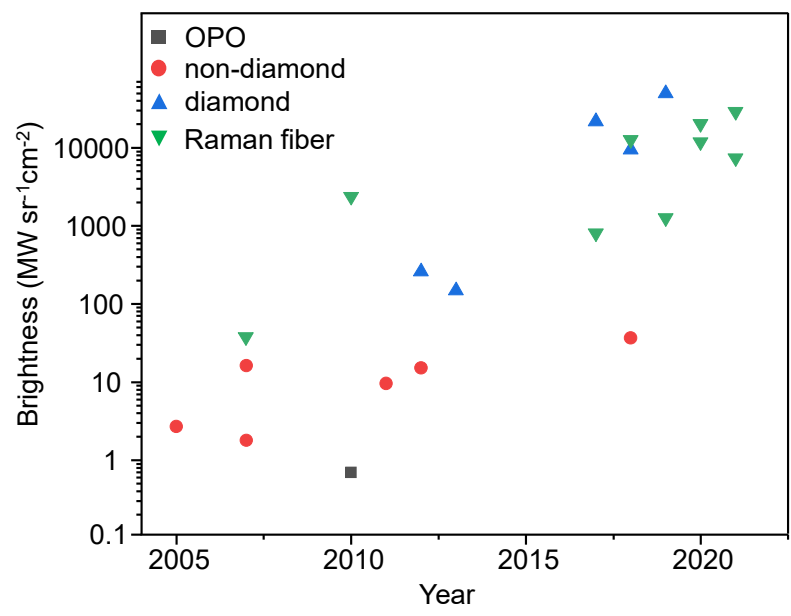

Figure 6. Brightness of CW solid-state nonlinear lasers over the past decade.

To the best of our knowledge, there is only one demonstration of brightness enhancement (BE) in case of CW OPO [133]. There has been multiple reports of BE in Raman lasers using various crystals $[51,52,59,106,121,123,129,138,139,141]$. It is clear from the figure that 
the Raman lasers have vastly improved the output beam quality, close to the diffractionlimit. It is also not surprising that DRLs have attained the highest BE among other crystalline media owing to its superior thermal properties. The brightness achieved by a DRL is more than three orders of magnitude than realized by a non-diamond Raman laser. On the other hand, Raman fiber lasers have displayed BE similar to DRLs [102,103,169,173-177]. However, in most of these cases, the thermal load in fibers with elevating output powers have prevented the beam quality to reach to near diffraction-limited output. For example, in ref. [102], the metalized fiber amplifier at $1130 \mathrm{~nm}$ exhibited a deterioration of $\mathrm{M}^{2}$ from 3.12 to 5.72 when the output power increased from $2.36 \mathrm{~kW}$ to the maximum power of $3.08 \mathrm{~kW}$. The maximum BEF of 27 has been achieved in a Raman GRIN fiber amplifier at $943 \mathrm{~W}$ at $1130 \mathrm{~nm}$ [175]. The $\mathrm{M}^{2}$ was reported to be 1.6 in this demonstration.

Based on these arguments, it is obvious that DRL technology offers a strong potential for power scalability as well as fulfill the additional requirement of preserving the beam quality. In many ways, diamond is the ideal laser material. Its extraordinarily high thermal conductivity of $2000 \mathrm{~W} / \mathrm{mK}$ (at room temperature)—highest among all known crystals, provides a unique opportunity to stretch the laser output power and brightness to levels not easily accessible by other Raman and OPO gain materials.

\section{Conclusions and Future Outlook}

We have reviewed the gain mechanisms, cavity configurations, power and brightness performance of OPOs and Raman lasers. Thermal loading in the gain crystal was a critical challenge in the power scaling of these lasers. However, in DRLs, this issue manifested only when output power exceeded the kilowatt level. They also exhibited the potential for extending the brightness range through their superior power handling capacity.

In conclusion, even though the OPOs are a mature nonlinear laser technology and more readily and commercially available, the path forward for power and brightness enhancement lies in the DRL technology.

Although the kilowatt demonstration of quasi-CW DRL witnessed thermal effects in the diamond crystal, the authors have not specified any material-cooling techniques in the paper. This raises an important question that is, how much power could be generated by the DRL with proper thermal management, before hitting the hard limits of thermal and mechanical stress in the crystal.

In the future, we envisage continuous operation of a DRL to generate output powers in multi-kilowatts. We believe the thermal lensing that can be anticipated in CW regime would be similar to what is observed in a crystal for quasi-CW operation. Therefore, we will study and tailor thermal management strategies for the laser; laser cooling mounts, cooling techniques and coolants (such as cryogenics, liquid cooling). We will investigate the various factors (such as thermal resistance, heat transfer coefficient) determining the rapid dissipation of thermal load from the crystal. We will also investigate and endeavor to enhance the heat transfer coefficients in the cooling pipes using twisted or coiled tubes, finning or tube inserts.

DRLs with multi-kW output powers with high brightness can pave the way for directed energy and remote sensing applications. Through intracavity frequency doubling, novel wavelengths with high power, good beam-quality, narrow-linewidth can be obtained in the visible spectrum. These wavelengths are useful in applications such as laser guide stars, medical therapy etc. Moreover, Raman cascading or appropriate choice of pump wavelengths allows a convenient means of extending the near-IR wavelengths to "eye-safe" and mid-IR spectral ranges. These wavelengths are particularly significant for LIDAR applications. Furthermore, the study of cooling techniques and architectures could be adapted to other Raman crystals with high damage threshold and moderate thermal conductivity, thus providing an alternative approach to diamond.

Author Contributions: Conceptualization and data curation, M.R. and S.S.; writing-original draft preparation, S.S.; writing-review and editing, M.R. and S.S.; visualization, supervision, funding acquisition M.R. All authors have read and agreed to the published version of the manuscript. 
Funding: This work was partially supported by a BRI- 5 contract on fundamental fiber laser science from AFOSR, by a grant from the University of Central Florida, and by funding from the State of Florida.

Data Availability Statement: Data sharing not applicable.

Conflicts of Interest: The authors declare no conflict of interest.

\section{References}

1. Geskus, D.; Jakutis-Neto, J.; Pask, H.M.; Wetter, N.U. Nonlinear Frequency Generation and Conversion: Materials, Devices, and Applications XIV; Ten Deep Blue to Cyan Emission Lines from an Intracavity Frequency Converted Raman Laser; International Society for Optics and Photonics: Bellingham, WA, USA, 2015.

2. Mildren, R.P.; Convery, M.; Pask, H.M.; Piper, J.A.; Mckay, T. Efficient, all-solid-state, Raman laser in the yellow, orange and red. Opt. Express 2004, 12, 785-790. [CrossRef]

3. Hollberg, L. Precision Spectroscopy, Diode Lasers, and Optical Frequency Measurement; CW Dye Lasers; Diane Publishing Co.: Collingdale, PA, USA, 2000; p. 105.

4. Miao, Z.W.; Yu, H.J.; Zhang, J.Y.; Zou, S.Z.; Zhao, P.F.; Lou, B.J.; Lin, X.C. Watt-level CW Ti: Sapphire oscillator directly pumped with green laser diodes module. IEEE Photonics Technol. Lett. 2020, 32, 247-250. [CrossRef]

5. Liu, H.; Sun, S.; Zheng, L.; Wang, G.; Tian, W.; Zhang, D.; Han, H.; Zhu, J.; Wei, Z. Review of laser-diode pumped Ti: Sapphire laser. Microw. Opt. Technol. Lett. 2021, 63, 2135-2144. [CrossRef]

6. M Squared SolsTiS. Available online: https://www.m2lasers.com/solstis.html (accessed on 24 October 2021).

7. Photonics Solutions Ltd. Available online: https://www.photonicsolutions.co.uk/upfiles/PrismSeriesIonLasersDatasheetLG1 1Jun18.pdf (accessed on 24 October 2021).

8. Anliker, P.; Luthi, H.; Seelig, W.; Steinger, J.; Weber, H.; Leutwyler, S.; Schumacher, E.; Woste, L. 33-W CW dye laser. IEEE J. Quantum Electron. 1977, 13, 547-548. [CrossRef]

9. Coherent INNOVA Series. Available online: https://www.coherent.com/lasers/ion/innova-series (accessed on 24 October 2021).

10. Gottwald, T.; Kuhn, V.; Schad, S.S.; Stolzenburg, C.; Killi, A. Technologies for Optical Countermeasures X; and High-Power Lasers 2013: Technology and Systems; Recent Developments in High Power Thin Disk Lasers at TRUMPF Laser; International Society for Optics and Photonics: Bellingham, WA, USA, 2013.

11. Stewen, C.; Contag, K.; Larionov, M.; Giesen, A.; Hugel, H. A 1-kW CW thin disc laser. IEEE J. Sel. Top. Quantum Electron. 2000, 6, 650-657. [CrossRef]

12. Wallace, J. High-Power Fiber Lasers: Kilowatt-Level Fiber Lasers Mature; Endeavor Business Media: Nashua, NH, USA, 2016.

13. Papastathopoulos, E.; Baumann, F.; Bocksrocker, O.; Gottwald, T.; Killi, A.; Metzger, B.; Schad, S.S.; Speker, N.; Ryba, T.; Zaske, S. Solid State Lasers XXX: Technology and Devices; High-Power High-brightness Disk Lasers for Advanced Applications; International Society for Optics and Photonics: Bellingham, WA, USA, 2021.

14. Jackson, S.D. Towards high-power mid-infrared emission from a fibre laser. Nat. Photonics 2012, 6, 423-431. [CrossRef]

15. Meleshkevich, M.; Platonov, N.; Gapontsev, D.; Drozhzhin, A.; Sergeev, V.; Gapontsev, V. The European Conference on Lasers and Electro-Optics; 415 W Single-Mode CW Thulium Fiber Laser in All-Fiber Format; Optical Society of America: Washington, DC, USA, 2007.

16. Lin, H.; Feng, Y.; Barua, P.; Sahu, J.K.; Nilsson, J. Advanced Solid State Lasers; 405 W Erbium-Doped Large-Core Fiber Laser; Optical Society of America: Washington, DC, USA, 2017.

17. Zhang, J.; Schulze, F.; Mak, K.F.; Pervak, V.; Bauer, D.; Sutter, D.; Pronin, O. High-power, high-efficiency Tm: YAG and Ho: YAG thin-disk lasers. Laser Photonics Rev. 2018, 12, 1700273. [CrossRef]

18. Ye, Y.; Yang, B.; Wang, P.; Zeng, L.; Xi, X.; Shi, C.; Zhang, H.; Wang, X.; Zhou, P.; Xu, X. Industrial 6 kW high-stability single-stage all-fiber laser oscillator based on conventional large mode area ytterbium-doped fiber. Laser Phys. 2021, 31, 035104. [CrossRef]

19. IPG Photonics Corporation. Available online: https://www.ipgphotonics.com/en/products/lasers/high-power-cw-fiber-lasers\# [1-micron] (accessed on 24 October 2021).

20. Hoffman, A.; Gmachl, C. Extending opportunities. Nat. Photonics 2012, 6, 407.

21. Wang, F.; Slivken, S.; Wu, D.H.; Lu, Q.Y.; Razeghi, M. Continuous wave quantum cascade lasers with $5.6 \mathrm{~W}$ output power at room temperature and 41\% wall-plug efficiency in cryogenic operation. AIP Adv. 2020, 10, 055120. [CrossRef]

22. Tabata, N.; Nagai, H.; Yoshida, H.; Hishii, M.; Tanaka, M.; Myoi, Y.; Akiba, T. Conference on Lasers and Electro-Optics; Industrial 20-kW cw CO 2 SAGE Laser; Optical Society of America: Washington, DC, USA, 1984.

23. Mid-Infrared Laser Sources. Available online: https://www.rp-photonics.com/mid_infrared_laser_sources.html (accessed on 12 September 2021).

24. Hudson, D.D. Short pulse generation in mid-IR fiber lasers. Opt. Fiber Technol. 2014, 20, 631-641. [CrossRef]

25. Yang, X.; Kitzler, O.; Spence, D.J.; Bai, Z.; Feng, Y.; Mildren, R.P. Diamond sodium guide star laser. Opt. Lett. 2020, 45, $1898-1901$. [CrossRef]

26. de Lima Ribeiro, A.; Pask, H. Remote Sensing of Natural Waters Using a Multichannel, Lidar-Compatible Raman Spectrometer and Blue Excitation. Front. Mar. Sci. 2020, 7, 43. [CrossRef] 
27. Yellow and Orange Lasers. Available online: https:/ / www.rp-photonics.com/yellow_and_orange_lasers.html (accessed on 12 March 2021).

28. Lux, O.; Sarang, S.; Kitzler, O.; Spence, D.J.; Mildren, R.P. Intrinsically stable high-power single longitudinal mode laser using spatial hole burning free gain. Optica 2016, 3, 876-881. [CrossRef]

29. Ahmed, S.A.; Mohsin, M.; Ali, S.M.Z. Survey and technological analysis of laser and its defense applications. Def. Technol. 2020, 17, 583-592. [CrossRef]

30. Paschotta, R. Laser Applications in the Encyclopedia of Laser Physics and Technology. Available online: https://www.rpphotonics.com/encyclopedia_cite.html?article=laser\%20applications (accessed on 24 October 2021).

31. Cousins, A.K. Temperature and thermal stress scaling in finite-length end-pumped laser rods. IEEE J. Quantum Electron. 1992, 28, 1057-1069. [CrossRef]

32. Tidwell, S.C.; Seamans, J.F.; Bowers, M.S.; Cousins, A.K. Scaling CW diode-end-pumped Nd: YAG lasers to high average powers. IEEE J. Quantum Electron. 1992, 28, 997-1009. [CrossRef]

33. Shukla, P.; Lawrence, J.; Zhang, Y. Understanding laser beam brightness: A review and new prospective in material processing. Opt. Laser Technol. 2015, 75, 40-51. [CrossRef]

34. Franken, E.P.; Hill, A.E.; Peters, C.W.; Weinreich, G. Generation of optical harmonics. Phys. Rev. Lett. 1961, 7, 118. [CrossRef]

35. Bass, M.; Franken, P.A.; Hill, A.E.; Peters, C.W.; Weinreich, G. Optical mixing. Phys. Rev. Lett. 1962, 8, 18. [CrossRef]

36. Guha, S.; Falk, J. The effects of focusing in the three-frequency parametric upconverter. J. Appl. Phys. 1980, 51, 50-60. [CrossRef]

37. Giordmaine, J.A.; Miller, R.C. Tunable coherent parametric oscillation in $\mathrm{LiNbO}_{3}$ at optical frequencies. Phys. Rev. Lett. 1965, 14, 973. [CrossRef]

38. Ammann, E.; Decker, C. 0.9-W Raman oscillator. J. Appl. Phys. 1977, 48, 1973-1975. [CrossRef]

39. Liao, Z.M.; Payne, S.A.; Dawson, W.J.; Drobshoff, A.D.; Ebbers, C.A.; Pennington, D.M.; Jovanovic, I.; Taylor, L.R. Nonlinear

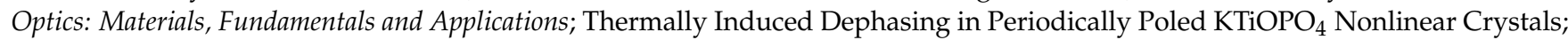
Optical Society of America: Washington, DC, USA, 2004.

40. Tovstonog, S.V.; Kurimura, S.; Suzuki, I.; Takeno, K.; Moriwaki, S.; Ohmae, N.; Mio, N.; Katagai, T. Thermal effects in high-power CW second harmonic generation in Mg-doped stoichiometric lithium tantalate. Opt. Express 2008, 16, 11294-11299. [CrossRef]

41. Kumar, S.C.; Samanta, G.K.; Ebrahim-Zadeh, M. High-power, single-frequency, continuous-wave second-harmonic-generation of ytterbium fiber laser in PPKTP and MgO: sPPLT. Opt. Express 2009, 17, 13711-13726. [CrossRef] [PubMed]

42. Černý, P.; Jelínková, H.; Zverev, P.G.; Basiev, T.T. Solid state lasers with Raman frequency conversion. Prog. Quantum Electron. 2004, 28, 113-143. [CrossRef]

43. Strößner, U.; Meyn, J.P.; Wallenstein, R.; Urenski, P.; Arie, A.; Rosenman, G.; Mlynek, J.; Schiller, S.; Peters, A. Single-frequency continuous-wave optical parametric oscillator system with an ultrawide tuning range of 550 to $2830 \mathrm{~nm}$. JOSA B 2002, 19, 1419-1424. [CrossRef]

44. Colville, F.G.; Henderson, A.J.; Padgett, M.J.; Zhang, J.; Dunn, M.H. Continuous-wave parametric oscillation in lithium triborate. Opt. Lett. 1993, 18, 205-207. [CrossRef]

45. Batchko, R.G.; Weise, D.; Plettner, T.; Miller, G.D.; Fejer, M.M.; Byer, R.L. CLEO'97. Summaries of Papers Presented at the Conference on Lasers and Electro-Optics; Continuous-Wave 532-nm-Pumped Singly Resonant Optical Parametric Oscillation in Periodically Poled Lithium Niobate; IEEE: Piscataway, NJ, USA, 1997.

46. Martinelli, M.; Zhang, K.S.; Coudreau, T.; Maître, A.; Fabre, C. Ultra-low threshold CW triply resonant OPO in the near infrared using periodically poled lithium niobate. J. Opt. A Pure Appl. Opt. 2001, 3, 300. [CrossRef]

47. Zeil, P.; Thilmann, N.; Pasiskevicius, V.; Laurell, F. High-power, single-frequency, continuous-wave optical parametric oscillator employing a variable reflectivity volume Bragg grating. Opt. Express 2014, 22, 29907-29913. [CrossRef]

48. Boyd, R.W. Nonlinear Optics; Academic Press: Cambridge, MA, USA, 2019.

49. Williams, R.J.; Kitzler, O.; Bai, Z.; Sarang, S.; Jasbeer, H.; McKay, A.; Antipov, S.; Sabella, A.; Lux, O.; Spence, D.J.; et al. High power diamond Raman lasers. IEEE J. Sel. Top. Quantum Electron. 2018, 24, 1-14. [CrossRef]

50. Yang, X.; Kitzler, O.; Spence, D.J.; Williams, R.J.; Bai, Z.; Sarang, S.; Zhang, L.; Feng, Y.; Mildren, R.P. Single-frequency 620 nm diamond laser at high power, stabilized via harmonic self-suppression and spatial-hole-burning-free gain. Opt. Lett. 2019, 44, 839-842. [CrossRef]

51. Casula, R.; Penttinen, J.P.; Guina, M.; Kemp, A.J.; Hastie, J.E. Cascaded crystalline Raman lasers for extended wavelength coverage: Continuous-wave, third-Stokes operation. Optica 2018, 5, 1406-1413. [CrossRef]

52. Antipov, S.; Sabella, A.; Williams, R.J.; Kitzler, O.; Spence, D.J.; Mildren, R.P. 1.2 kW quasi-steady-state diamond Raman laser pumped by an $\mathrm{M}^{2}=15$ beam. Opt. Lett. 2019, 44, 2506-2509. [CrossRef]

53. Demtröder, W. Laser Spectroscopy 1: Basic Principles; Optical Parametric Oscillators; Springer: Berlin, Germany, 2014.

54. Mildren, R.; Rabeau, J. Optical Engineering of Diamond; Diamond Raman Laser Design and Performance; John Wiley \& Sons: Hoboken, NJ, USA, 2013.

55. Dunn, M.H.; Stothard, D. Intra-Cavity Optical Parametric Oscillator. US 8,867,584 B2 21 October 2014.

56. Laurell, F. Nonlinear Optics Continued-Optical Parametric Oscillators and Amplifiers. 2013. Available online: http://indico.ictp. it/event/a12164/session/18/contribution/11/material/0/0.pdf (accessed on 21 February 2021).

57. Ebrahim-Zadeh, M. Handbook of Optics; Continuous-Wave Optical Parametric Oscillators; McGraw-Hill: New York, NY, USA, 2010; pp. 1-33. 
58. Ferrara, M.A.; Sirleto, L. Integrated Raman Laser: A Review of the Last Two Decades. Micromachines 2020, 11, 330. [CrossRef] [PubMed]

59. Bai, Z.; Williams, R.J.; Jasbeer, H.; Sarang, S.; Kitzler, O.; Mckay, A.; Mildren, R.P. Large brightness enhancement for quasicontinuous beams by diamond Raman laser conversion. Opt. Lett. 2018, 43, 563-566. [CrossRef] [PubMed]

60. Williams, R.J.; Kitzler, O.; McKay, A.; Mildren, R.P. Investigating diamond Raman lasers at the $100 \mathrm{~W}$ level using quasi-continuouswave pumping. Opt. Lett. 2014, 39, 4152-4155. [CrossRef] [PubMed]

61. Henderson, A.; Esquinasi, P. Fiber Lasers VII: Technology, Systems, and Applications; 23-Watt 77\% Efficient CW OPO Pumped by a Fiber Laser; International Society for Optics and Photonics: Bellingham, WA, USA, 2010.

62. Kumar, S.C.; Das, R.; Samanta, G.K.; Ebrahim-Zadeh, M. Optimally-output-coupled, 17.5 W, fiber-laser-pumped continuous-wave optical parametric oscillator. Appl. Phys. B 2011, 102, 31-35.

63. Smith, R.G.; Geusic, J.E.; Levinstein, H.J.; Rubin, J.J.; Singh, S.; Van Uitert, L.G. Continuous optical parametric oscillation in $\mathrm{Ba}_{2} \mathrm{NaNb}_{5} \mathrm{O}_{15}$. Appl. Phys. Lett. 1968, 12, 308-310. [CrossRef]

64. Smith, R.; Parker, J. Experimental observation of and comments on optical parametric oscillation internal to the laser cavity. $J$. Appl. Phys. 1970, 41, 3401-3408. [CrossRef]

65. Byer, R.L.; Oshman, M.K.; Young, J.F.; Harris, S.E. Visible CW parametric oscillator. Appl. Phys. Lett. 1968, 13, 109-111. [CrossRef]

66. Byer, R.; Kovrigin, A.; Young, J. A cw ring-cavity parametric oscillator. Appl. Phys. Lett. 1969, 15, 136-137. [CrossRef]

67. Sperling, J.; Hens, K. ; Tunable Laser Light Sources Advance Nanophotonics Research; Photonics Media: Pittsfield, MA, USA, 2018.

68. Devi, K. Continuous-Wave Optical Parametric Oscillators and Frequency Conversion Sources from the Ultraviolet to the Mid-Infrared. Ph.D. Thesis, Universitat Politècnica de Catalunya, Barcelona, Spain, 24 October 2013.

69. Gerstenberger, D.; Wallace, R.W. Continuous-wave operation of a doubly resonant lithium niobate optical parametric oscillator system tunable from 966 to $1185 \mathrm{~nm}$. JOSA B 1993, 10, 1681-1683. [CrossRef]

70. Colville, F.; Padgett, M.; Dunn, M. Continuous-wave, dual-cavity, doubly resonant, optical parametric oscillator. Appl. Phys. Lett. 1994, 64, 1490-1492. [CrossRef]

71. Colville, F.G.; Padgett, M.J.; Henderson, A.J.; Zhang, J.; Dunn, M.H. Continuous-wave parametric oscillator pumped in the ultraviolet. Opt. Lett. 1993, 18, 1065-1067. [CrossRef]

72. Kozlovsky, W.J.; Nabors, C.D.; Eckardt, R.C.; Byer, R.L. Monolithic MgO: $\mathrm{LiNbO}_{3}$ doubly resonant optical parametric oscillator pumped by a frequency-doubled diode-laser-pumped Nd: YAG laser. Opt. Lett. 1989, 14, 66-68. [CrossRef]

73. Lee, D.; Wong, N. Stabilization and tuning of a doubly resonant optical parametric oscillator. JOSA B 1993, 10, 1659-1667. [CrossRef]

74. Bosenberg, W.R.; Drobshoff, A.; Alexander, J.I.; Myers, L.E.; Byer, R.L. Continuous-wave singly resonant optical parametric oscillator based on periodically poled $\mathrm{LiNbO}_{3}$. Opt. Lett. 1996, 21, 713-715. [CrossRef]

75. Eckardt, R.C.; Nabors, C.D.; Kozlovsky, W.J.; Byer, R.L. Optical parametric oscillator frequency tuning and control. JOSA B 1991, 8, 646-667. [CrossRef]

76. Yang, S.; Eckardt, R.; Byer, R. Continuous-wave singly resonant optical parametric oscillator pumped by a single-frequency resonantly doubled Nd: YAG laser. Opt. Lett. 1993, 18, 971-973. [CrossRef]

77. Samanta, G.; Ebrahim-Zadeh, M. Continuous-wave singly-resonant optical parametric oscillator with resonant wave coupling. Opt. Express 2008, 16, 6883-6888. [CrossRef]

78. Edwards, T.J.; Turnbull, G.A.; Dunn, M.H.; Ebrahimzadeh, M. Continuous-wave, singly-resonant, optical parametric oscillator based on periodically poled $\mathrm{KTiOPO}_{4}$. Opt. Express 2000, 6, 58-63. [CrossRef]

79. Bosenberg, W.R.; Drobshoff, A.; Alexander, J.I.; Myers, L.E.; Byer, R.L. 93\% pump depletion, 3.5-W continuous-wave, singly resonant optical parametric oscillator. Opt. Lett. 1996, 21, 1336-1338. [CrossRef]

80. Lindsay, I.D.; Adhimoolam, B.; Gross, P.; Klein, M.E.; Boller, K.J. 110GHz rapid, continuous tuning from an optical parametric oscillator pumped by a fiber-amplified DBR diode laser. Opt. Express 2005, 13, 1234-1239. [CrossRef]

81. Tan, W.; Liu, Z.; Cong, Z.; Zhao, Z.; Qin, Z.; Chen, X.; Yang, Z.; Rao, H.; Zhao, E.; Zhang, S.; et al. 4.4 W, Continuous-Wave, Narrow Linewidth Optical Parametric Oscillator at $3.77 \mu \mathrm{m}$. IEEE Photonics Technol. Lett. 2019, 31, 1131-1134. [CrossRef]

82. Vainio, M.; Peltola, J.; Persijn, S.; Harren, F.J.; Halonen, L. Singly resonant cw OPO with simple wavelength tuning. Opt. Express 2008, 16, 11141-11146. [CrossRef]

83. Klein, M.E.; Laue, C.K.; Lee, D.H.; Boller, K.J.; Wallenstein, R. Diode-pumped singly resonant continuous-wave optical parametric oscillator with wide continuous tuning of the near-infrared idler wave. Opt. Lett. 2000, 25, 490-492. [CrossRef]

84. Samanta, G.; Fayaz, G.; Ebrahim-Zadeh, M. 1.59 W, single-frequency, continuous-wave optical parametric oscillator based on MgO: sPPLT. Opt. Lett. 2007, 32, 2623-2625. [CrossRef]

85. Henderson, A.; Stafford, R. Intra-cavity power effects in singly resonant cw OPOs. Appl. Phys. B 2006, 85, 181-184. [CrossRef]

86. Shukla, M.K.; Das, R. High-power single-frequency source in the mid-infrared using a singly resonant optical parametric oscillator pumped by Yb-fiber laser. IEEE J. Sel. Top. Quantum Electron. 2017, 24, 1-6. [CrossRef]

87. Mieth, S.; Henderson, A.; Halfmann, T. Tunable, continuous-wave optical parametric oscillator with more than $1 \mathrm{~W}$ output power in the orange visible spectrum. Opt. Express 2014, 22, 11182-11191. [CrossRef]

88. Vainio, M.; Ozanam, C.; Ulvila, V.; Halonen, L. Tuning and stability of a singly resonant continuous-wave optical parametric oscillator close to degeneracy. Opt. Express 2011, 19, 22515-22527. [CrossRef] 
89. Vainio, M.; Peltola, J.; Persijn, S.; Harren, F.J.M.; Halonen, L. Thermal effects in singly resonant continuous-wave optical parametric oscillators. Appl. Phys. B 2009, 94, 411. [CrossRef]

90. Yu, C.X.; Augst, S.J.; Redmond, S.M.; Goldizen, K.C.; Murphy, D.V.; Sanchez, A.; Fan, T.Y. Coherent combining of a 4 kW, eight-element fiber amplifier array. Opt. Lett. 2011, 36, 2686-2688. [CrossRef]

91. Odier, A.; Chtouki, R.; Bourdon, P.; Melkonian, J.M.; Lombard, L.; Lefebvre, M.; Durécu, A. Coherent combining of mid-infrared difference frequency generators. Opt. Lett. 2019, 44, 566-569. [CrossRef]

92. Chtouki, R.; Bourdon, P.; Durécu, A.; Lombard, L.; Planchat, C.; Raybaut, M.; Godard, A. Nonlinear Frequency Generation and Conversion: Materials and Devices XIX; An Experimental Demonstration of Coherent Combining Applied to Optical Parametric Oscillators; International Society for Optics and Photonics: Bellingham, WA, USA, 2020.

93. Marx, R.E.; Hubner, U.; Abdul-Halim, I.B.; Heppner, J.O.; Ni, Y.C.; Willenberg, G.; Weiss, C. Far-infrared CW Raman and laser gain of $14 \mathrm{NH}_{3}$. IEEE J. Quantum Electron. 1981, 17, 1123-1127. [CrossRef]

94. Jabr, S.N. Gain and noise characteristics of a continuous-wave Raman laser. Opt. Lett. 1987, 12, 690-692. [CrossRef]

95. Brasseur, J.K.; Repasky, K.S.; Carlsten, J.L. Continuous-wave Raman laser in H2. Opt. Lett. 1998, 23, 367-369. [CrossRef] [PubMed]

96. Zhang, L.; Liu, C.; Jiang, H.; Qi, Y.; He, B.; Zhou, J.; Gu, X.; Feng, Y. Kilowatt ytterbium-Raman fiber laser. Opt. Express 2014, 22, 18483-18489. [CrossRef]

97. Xiao, Q.; Yan, P.; Li, D.; Sun, J.; Wang, X.; Huang, Y.; Gong, M. Bidirectional pumped high power Raman fiber laser. Opt. Express 2016, 24, 6758-6768. [CrossRef]

98. Supradeepa, V.; Nicholson, J.W. Power scaling of high-efficiency $1.5 \mu \mathrm{m}$ cascaded Raman fiber lasers. Opt. Lett. 2013, 38, 2538-2541. [CrossRef]

99. Pask, H.M. The design and operation of solid-state Raman lasers. Prog. Quantum Electron. 2003, 27, 3-56. [CrossRef]

100. Hill, K.; Kawasaki, B.; Johnson, D. Low-threshold cw Raman laser. Appl. Phys. Lett. 1976, 29, 181-183. [CrossRef]

101. Chen, Y.; Yao, T.; Leng, J.; Xiao, H. $1.6 \mathrm{~kW}$ Raman fiber amplifier based on multimode graded index fiber. In Proceedings of the 2019 18th International Conference on Optical Communications and Networks (ICOCN), Huangshan, China, 5-8 August 2019; IEEE: Piscataway, NJ, USA, 2019.

102. Chen, Y.; Yao, T.; Xiao, H.; Leng, J.; Zhou, P. $3 \mathrm{~kW}$ passive-gain-enabled metalized Raman fiber amplifier with brightness enhancement. J. Light. Technol. 2021, 39, 1785-1790. [CrossRef]

103. Glick, Y.; Shamir, Y.; Aviel, M.; Sintov, Y.; Goldring, S.; Shafir, N.; Pearl, S. 1.2 kW clad pumped Raman all-passive-fiber laser with brightness enhancement. Opt. Lett. 2018, 43, 4755-4758. [CrossRef]

104. Supradeepa, V.R.; Nicholson, J.W.; Headley, C.; Lee, Y.W.; Palsdottir, B.; Jakobsen, D. Fiber Lasers IX: Technology, Systems, and Applications; Cascaded Raman Fiber Laser at $1480 \mathrm{~nm}$ with Output Power of $104 \mathrm{~W}$; International Society for Optics and Photonics: Bellingham, WA, USA, 2012.

105. Grabtchikov, A.S.; Lisinetskii, V.A.; Orlovich, V.A.; Schmitt, M.; Maksimenka, R.; Kiefer, W. Multimode pumped continuous-wave solid-state Raman laser. Opt. Lett. 2004, 29, 2524-2526. [CrossRef]

106. Demidovich, A.A.; Grabtchikov, A.S.; Lisinetskii, V.A.; Burakevich, V.N.; Orlovich, V.A.; Kiefer, W. Continuous-wave Raman generation in a diode-pumped $\mathrm{Nd}^{3+}: \mathrm{KGd}\left(\mathrm{WO}_{4}\right)_{2}$ laser. Opt. Lett. 2005, 30, 1701-1703. [CrossRef] [PubMed]

107. Lin, J.; Pask, H. Nd: GdVO 4 self-Raman laser using double-end polarised pumping at $880 \mathrm{~nm}$ for high power infrared and visible output. Appl. Phys. B 2012, 108, 17-24. [CrossRef]

108. Fan, L.; Zhao, W.; Qiao, X.; Xia, C.; Wang, L.; Fan, H.; Shen, M. An efficient continuous-wave $\mathrm{YVO}_{4} / \mathrm{Nd} \mathrm{YVO}_{4} / \mathrm{YVO}_{4}$ self-Raman laser pumped by a wavelength-locked $878.9 \mathrm{~nm}$ laser diode. Chin. Phys. B 2016, 25, 114207. [CrossRef]

109. Fan, L.; Fan, Y.X.; Duan, Y.H.; Wang, Q.; Wang, H.T.; Jia, G.H.; Tu, C.Y. Continuous-wave intracavity Raman laser at $1179.5 \mathrm{~nm}$ with $\mathrm{SrWO}_{4}$ Raman crystal in diode-end-pumped Nd: $\mathrm{YVO}_{4}$ laser. Appl. Phys. B 2009, 94, 553-557. [CrossRef]

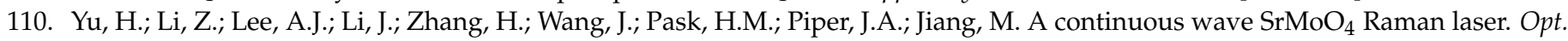
Lett. 2011, 36, 579-581. [CrossRef]

111. Pask, H.M. Continuous-wave, all-solid-state, intracavity Raman laser. Opt. Lett. 2005, 30, 2454-2456. [CrossRef]

112. Spence, D.J.; Dekker, P.; Pask, H.M. Modeling of continuous wave intracavity Raman lasers. IEEE J. Sel. Top. Quantum Electron. 2007, 13, 756-763. [CrossRef]

113. Bonner, G.M.; Lin, J.; Kemp, A.J.; Wang, J.; Zhang, H.; Spence, D.J.; Pask, H.M. Spectral broadening in continuous-wave intracavity Raman lasers. Opt. Express 2014, 22, 7492-7502. [CrossRef] [PubMed]

114. Kitzler, O.; McKay, A.; Mildren, R.P. Continuous-wave wavelength conversion for high-power applications using an external cavity diamond Raman laser. Opt. Lett. 2012, 37, 2790-2792. [CrossRef]

115. Lux, O.; Sarang, S.; Williams, R.J.; McKay, A.; Mildren, R.P. Single longitudinal mode diamond Raman laser in the eye-safe spectral region for water vapor detection. Opt. Express 2016, 24, 27812-27820. [CrossRef]

116. Williams, R.J.; Nold, J.; Strecker, M.; Kitzler, O.; McKay, A.; Schreiber, T.; Mildren, R.P. Efficient Raman frequency conversion of high-power fiber lasers in diamond. Laser Photonics Rev. 2015, 9, 405-411. [CrossRef]

117. Antipov, S.; Williams, R.J.; Sabella, A.; Kitzler, O.; Berhane, A.; Spence, D.J.; Mildren, R.P. Analysis of a thermal lens in a diamond Raman laser operating at $1.1 \mathrm{~kW}$ output power. Opt. Express 2020, 28, 15232-15239. [CrossRef]

118. Lubeigt, W.; Bonner, G.M.; Hastie, J.E.; Dawson, M.D.; Burns, D.; Kemp, A.J. Continuous-wave diamond Raman laser. Opt. Lett. 2010, 35, 2994-2996. [CrossRef] 
119. Orlovich, V.A.; Burakevich, V.N.; Grabtchikov, A.S.; Lisinetskii, V.A.; Demidovich, A.A.; Eichler, H.J.; Turpin, P.Y. Continuouswave intracavity Raman generation in $\mathrm{PbWO}_{4}$ crystal in the $\mathrm{Nd}$ : $\mathrm{YVO}_{4}$ laser. Laser Phys. Lett. 2005, 3, 71. [CrossRef]

120. Dekker, P.; Pask, H.M.; Piper, J.A. All-solid-state $704 \mathrm{~mW}$ continuous-wave yellow source based on an intracavity, frequencydoubled crystalline Raman laser. Opt. Lett. 2007, 32, 1114-1116. [CrossRef]

121. Parrotta, D.C.; Kemp, A.J.; Dawson, M.D.; Hastie, J.E. Multiwatt, continuous-wave, tunable diamond Raman laser with intracavity frequency-doubling to the visible region. IEEE J. Sel. Top. Quantum Electron. 2013, 19, 1400108. [CrossRef]

122. Fan, L.; Fan, Y.X.; Li, Y.Q.; Zhang, H.; Wang, Q.; Wang, J.; Wang, H.T. High-efficiency continuous-wave Raman conversion with a $\mathrm{BaWO}_{4}$ Raman crystal. Opt. Lett. 2009, 34, 1687-1689. [CrossRef] [PubMed]

123. Savitski, V.G.; Friel, I.; Hastie, J.E.; Dawson, M.D.; Burns, D.; Kemp, A.J. Characterization of single-crystal synthetic diamond for multi-watt continuous-wave Raman lasers. IEEE J. Quantum Electron. 2011, 48, 328-337. [CrossRef]

124. Chang, M.T.; Zhuang, W.Z.; Su, K.W.; Yu, Y.T.; Chen, Y.F. Efficient continuous-wave self-Raman Yb:KGW laser with a shift of $89 \mathrm{~cm}^{-1}$. Opt. Express 2013, 21, 24590-24598. [CrossRef]

125. Kores, C.C.; Jakutis-Neto, J.; Geskus, D.; Pask, H.M.; Wetter, N.U. Diode-side-pumped continuous wave $\mathrm{Nd}^{3+}: \mathrm{YVO}_{4}$ self-Raman laser at $1176 \mathrm{~nm}$. Opt. Lett. 2015, 40, 3524-3527. [CrossRef]

126. Ferreira, M.S.; Wetter, N. Yb:KGW self-Raman laser with $89 \mathrm{~cm}^{-1}$ Stokes shift and more than $32 \%$ diode-to-Stokes optical efficiency. Opt. Laser Technol. 2020, 121, 105835. [CrossRef]

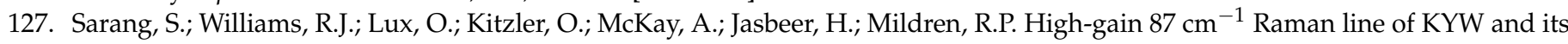
impact on continuous-wave Raman laser operation. Opt. Express 2016, 24, 21463-21473. [CrossRef] [PubMed]

128. Williams, R.J.; Spence, D.J.; Lux, O.; Mildren, R.P. High-power continuous-wave Raman frequency conversion from $1.06 \mu \mathrm{m}$ to $1.49 \mu \mathrm{m}$ in diamond. Opt. Express 2017, 25, 749-757. [CrossRef]

129. Bai, Z.; Williams, R.J.; Jasbeer, H.; Sarang, S.; McKay, A.; Mildren, R.P. Brightness enhancement of continuous-wave beams using a diamond Raman laser. In Proceedings of the Conference on Lasers and Electro-Optics/Pacific Rim, Singapore, 31 July-4 August 2017; Optical Society of America: Washington, DC, USA, 2017.

130. McKay, A.; Kitzler, O.; Mildren, R.P. Simultaneous brightness enhancement and wavelength conversion to the eye-safe region in a high-power diamond Raman laser. Laser Photonics Rev. 2014, 8, L37-L41. [CrossRef]

131. Marshall, L.R.; Kaz, A.; Aytur, O. Multimode pumping of optical parametric oscillators. IEEE J. Quantum Electron. 1996, 32, 177-182. [CrossRef]

132. Van Herpen, M.M.J.W.; Li, S.; Bisson, S.E.; te Lintel Hekkert, S.; Harren, F.J.M. Tuning and stability of a continuous-wave mid-infrared high-power single resonant optical parametric oscillator. Appl. Phys. B 2002, 75, 329-333. [CrossRef]

133. Nieuwenhuis, A.F.; Lee, C.J.; Sumpf, B.; van der Slot, P.J.; Erbert, G.; Boller, K.J. One-Watt level mid-IR output, singly resonant, continuous-wave optical parametric oscillator pumped by a monolithic diode laser. Opt. Express 2010, 18, 11123-11131. [CrossRef] [PubMed]

134. Ehrlich, Y.; Pearl, S.; Peleg, G.; Fastig, S. High Brightness Tunable OPO Source for 8-12 $\mu$ m Dial. In Proceedings of the 22nd Internation Laser Radar Conference (ILRC 2004), Matera City, Basilicata, Italy, 12-16 July 2004.

135. Chang, R.; Lehmberg, R.; Duignan, M.; Djeu, N. Raman beam cleanup of a severely aberrated pump laser. IEEE J. Quantum Electron. 1985, 21, 477-487. [CrossRef]

136. Chang, R.S.; Duignan, M.T.; Lehmberg, R.H.; Djeu, N. Excimer Lasers: Their Applications \& New Frontiers in Lasers; Use of Stimulated Raman Scattering for Reducing the Divergence of Severely Aberrated Laser Beams; International Society for Optics and Photonics: Bellingham, WA, USA, 1984.

137. Partanen, J.; Shaw, M. High-power forward Raman amplifiers employing low-pressure gases in light guides. I. Theory and applications. JOSA B 1986, 3, 1374-1389. [CrossRef]

138. Dekker, P.; Pask, H.M.; Spence, D.J.; Piper, J.A. Continuous-wave, intracavity doubled, self-Raman laser operation in Nd:GdVO 4 at $586.5 \mathrm{~nm}$. Opt. Express 2007, 15, 7038-7046. [CrossRef] [PubMed]

139. Parrotta, D.C.; Lubeigt, W.; Kemp, A.J.; Burns, D.; Dawson, M.D.; Hastie, J.E. Continuous-wave Raman laser pumped within a semiconductor disk laser cavity. Opt. Lett. 2011, 36, 1083-1085. [CrossRef]

140. Casula, R.; Penttinen, J.P.; Kemp, A.J.; Guina, M.; Hastie, J.E. 1.4 mm continuous-wave diamond Raman laser. Opt. Express 2017, 25, 31377-31383. [CrossRef]

141. Burakevich, V.N.; Lisinetskii, V.A.; Grabtchikov, A.S.; Demidovich, A.A.; Orlovich, V.A.; Matrosov, V.N. Diode-pumped continuous-wave Nd: $\mathrm{YVO}_{4}$ laser with self-frequency Raman conversion. Appl. Phys. B 2007, 86, 511-514. [CrossRef]

142. Lee, A.J.; Pask, H.M.; Omatsu, T.; Dekker, P.; Piper, J.A. All-solid-state continuous-wave yellow laser based on intracavity frequency-doubled self-Raman laser action. Appl. Phys. B 2007, 88, 539-544. [CrossRef]

143. Bai, Z.; Williams, R.J.; Kitzler, O.; Sarang, S.; Spence, D.J.; Mildren, R.P. 302 W quasi-continuous cascaded diamond Raman laser at 1.5 microns with large brightness enhancement. Opt. Express 2018, 26, 19797-19803. [CrossRef] [PubMed]

144. Harris, S.E. Tunable optical parametric oscillators. Proc. IEEE 1969, 57, 2096-2113. [CrossRef]

145. Chen, Y.F.; Pan, Y.Y.; Liu, Y.C.; Cheng, H.P.; Tsou, C.H.; Liang, H.C. Efficient high-power continuous-wave lasers at green-limeyellow wavelengths by using a Nd: $\mathrm{YVO}_{4}$ self-Raman crystal. Opt. Express 2019, 27, 2029-2035. [CrossRef]

146. Geskus, D.; Neto, J.J.; Pask, H.M.; Wetter, N.U. CLEO: QELS Fundamental Science; Two New Blue Laser Emission Lines from an Intracavity Raman Laser; Optical Society of America: Washington, DC, USA, 2014. 
147. Pask, H.M.; Dekker, P.; Mildren, R.P.; Spence, D.J.; Piper, J.A. Wavelength-versatile visible and UV sources based on crystalline Raman lasers. Prog. Quantum Electron. 2008, 32, 121-158. [CrossRef]

148. Myers, L.E.; Bosenberg, W.R. Periodically poled lithium niobate and quasi-phase-matched optical parametric oscillators. IEEE J. Quantum Electron. 1997, 33, 1663-1672. [CrossRef]

149. Zhao, H.; Lima, I.; Major, A. Near-infrared properties of periodically poled $\mathrm{KTiOPO}_{4}$ and stoichiometric $\mathrm{MgO}^{-d o p e d ~} \mathrm{LiTaO}_{3}$ crystals for high power optical parametric oscillation with femtosecond pulses. Laser Phys. 2010, 20, 1404-1409. [CrossRef]

150. Robertson, G. Optical Parametric Oscillators: A Comparison of New Materials. Ph.D. Thesis, University of St. Andrews, St. Andrews, Scotland, 1993.

151. Akbari, R.; Major, A. Optical, spectral and phase-matching properties of BIBO, BBO and LBO crystals for Opticalparametric oscillation in the visible and near-infrared wavelength ranges. Laser Phys. 2013, 23, 035401. [CrossRef]

152. Bierlein, J.D.; Vanherzeele, H. Potassium titanyl phosphate: Properties and new applications. JOSA B 1989, 6, 622-633. [CrossRef]

153. Hellström, J. Nanosecond Optical Parametric Oscillators and Amplifiers Based on Periodically Poled KTiOPO 4 . Ph.D. Thesis, Fysiska Institutionen, Lund, Sweden, 2001.

154. Karlsson, H. Fabrication of Periodically Poled Crystals from the KTP Family and Their Applications in Nonlinear Optics. Ph.D. Thesis, Fysiska Institutionen, Lund, Sweden, 1999.

155. Lithium Niobate Properties. Available online: http://www.azurephotonicsus.com/material/crystals/Lithium\%20Niobate\%20 crystal.html (accessed on 21 February 2021).

156. Piper, J.A.; Pask, H.M. Crystalline Raman lasers. IEEE J. Sel. Top. Quantum Electron. 2007, 13, 692-704. [CrossRef]

157. Linares, R.; Doering, P. Properties of large single crystal diamond. Diam. Relat. Mater. 1999, 8, 909-915. [CrossRef]

158. Friel, I.; Geoghegan, S.L.; Twitchen, D.J.; Scarsbrook, G.A. Optics and Photonics for Counterterrorism and Crime Fighting VI and Optical Materials in Defence Systems Technology VII; Development of High Quality Single Crystal Diamond for Novel Laser Applications; International Society for Optics and Photonics: Bellingham, WA, USA, 2010.

159. Martineau, P.M.; Gaukroger, M.P.; Guy, K.B.; Lawson, S.C.; Twitchen, D.J.; Friel, I.; Hansen, J.O.; Summerton, G.C.; Addison, T.P.G.; Burns, R. High crystalline quality single crystal chemical vapour deposition diamond. J. Condens. Matter Phys. 2009, 21, 364205. [CrossRef]

160. Romanyuk, Y. Liquid-Phase Epitaxy of Doped $\mathrm{KY}\left(\mathrm{WO}_{4}\right)_{2}$ Layers for Waveguide Lasers. Ph.D. Thesis, EPFL, Lausanne, Switzerland, 2005.

161. Kaminskii, A.A.; Konstantinova, A.F.; Orekhova, V.P.; Butashin, A.V.; Klevtsova, R.F.; Pavlyuk, A.A. Optical and nonlinear laser properties of the $\chi(3)$-active monoclinic $\alpha-\mathrm{KY}\left(\mathrm{WO}_{4}\right)_{2}$ crystals. Crystallogr. Rep. 2001, 46, 665-672. [CrossRef]

162. Sabella, A.; Spence, D.J.; Mildren, R.P. Pump-probe measurements of the Raman gain coefficient in crystals using multilongitudinal-mode beams. IEEE J. Quantum Electron. 2015, 51, 1-8. [CrossRef]

163. Basiev, T.T.; Osiko, V.V.; Prokhorov, A.M.; Dianov, E.M. Topics in Applied Physics; Crystalline and Fiber Raman Lasers. Solid-State Mid-Infrared Laser Sources; Springer: Berlin, Germany, 2003; Volume 89.

164. Denker, B.; Shklovsky, E. Handbook of Solid-State Lasers: Materials, Systems and Applications; Elsevier: Amsterdam, The Netherlands, 2013.

165. Lowenthal, D.D. Solid State Lasers VIII; CW Periodically Poled $\mathrm{LiNbO}_{3}$ Optical Parametric Oscillator Model with Strong Idler Absorption; International Society for Optics and Photonics: Bellingham, WA, USA, 1999.

166. Grubb, S.G.; Erdogan, T.; Mizrahi, V.; Strasser, T.; Cheung, W.Y.; Reed, W.A.; Lemaire, P.J.; Miller, A.E.; Kosinski, S.G.; Nykolak, G.; et al. Optical Amplifiers and Their Applications; 1.3 m Cascaded Raman Amplifier in Germanosilicate Fibers; Optical Society of America: Breckenridge, CO, USA, 1994.

167. Grubb, S.G.; Strasser, T.; Cheung, W.Y.; Reed, W.A.; Mizrahi, V.; Erdogan, T.; Lemaire, P.J.; Vengsarkar, A.M.; DiGiovanni, D.J.; Peckham, D.W.; et al. Optical Amplifiers and Their Applications; High-Power 1.48 um Cascaded Raman Laser in Germanosilicate Fibers; Optical Society of America: Davos, Switzerland, 1995.

168. Codemard, C.A.; Dupriez, P.; Jeong, Y.; Sahu, J.K.; Ibsen, M.; Nilsson, J. High-power continuous-wave cladding-pumped Raman fiber laser. Opt. Lett. 2006, 31, 2290-2292. [CrossRef] [PubMed]

169. Codemard, C.A.; Ji, J.; Sahu, J.K.; Nilsson, J. Fiber Lasers VII: Technology, Systems, and Applications; 100-W CW Cladding-Pumped Raman Fiber Laser at 1120 nm; International Society for Optics and Photonics: Bellingham, WA, USA, 2010.

170. Feng, Y.; Taylor, L.R.; Calia, D.B. 150 W highly-efficient Raman fiber laser. Opt. Express 2009, 17, 23678-23683. [CrossRef] [PubMed]

171. Glick, Y.; Fromzel, V.; Zhang, J.; Dahan, A.; Ter-Gabrielyan, N.; Pattnaik, R.K.; Dubinskii, M. High power, high efficiency diode pumped Raman fiber laser. Laser Phys. Lett. 2016, 13, 065101. [CrossRef]

172. Glick, Y.; Fromzel, V.; Zhang, J.; Ter-Gabrielyan, N.; Dubinskii, M. High-efficiency, 154 W CW, diode-pumped Raman fiber laser with brightness enhancement. Appl. Opt. 2017, 56, B97-B102. [CrossRef] [PubMed]

173. Chen, Y.; Yao, T.; Xiao, H.; Leng, J.; Zhou, P. Greater than $2 \mathrm{~kW}$ all-passive fiber Raman amplifier with good beam quality. High Power Laser Sci. Eng. 2020, 8, e33. [CrossRef]

174. Chen, Y.; Yao, T.; Xiao, H.; Leng, J.; Zhou, P. High-power cladding pumped Raman fiber amplifier with a record beam quality. Opt. Lett. 2020, 45, 2367-2370. [CrossRef]

175. Fan, C.; Xiao, H.; Yao, T.; Xu, J.; Chen, Y.; Leng, J.; Zhou, P. Kilowatt level Raman amplifier based on $100 \mu$ m core diameter multimode GRIN fiber with $\mathrm{M}^{2}=1.6$. Opt. Lett. 2021, 46, 3432-3435. [CrossRef] 
176. Zlobina, E.A.; Kablukov, S.I.; Wolf, A.A.; Nemov, I.N.; Dostovalov, A.V.; Tyrtyshnyy, V.A.; Myasnikov, D.V.; Babin, S.A. Generating high-quality beam in a multimode LD-pumped all-fiber Raman laser. Opt. Express 2017, 25, 12581-12587. [CrossRef] [PubMed]

177. Terry, N.B.; Engel, K.T.; Alley, T.G.; Russell, T.H. Use of a continuous wave Raman fiber laser in graded-index multimode fiber for SRS beam combination. Opt. Express 2007, 15, 602-607. [CrossRef] [PubMed] 